\title{
Analysis of liquid-to-gas mass transfer, mixing and hydrogen production in
}

\section{dark fermentation process}

\author{
B. Chezeau ${ }^{1,2}$, J.P. Fontaine ${ }^{1,2}$, Ch. Vial ${ }^{1,2}$ \\ ${ }^{1}$ Université Clermont Auvergne, CNRS, SIGMA Clermont, Institut Pascal, F-63000 CLERMONT-FERRAND,
}

FRANCE

${ }^{2}$ Université Clermont Auvergne, LabEx IMobS ${ }^{3}$, F-63000 CLERMONT-FERRAND, FRANCE

\begin{abstract}
The aim of this work was to investigate mixing and liquid-to-gas mass transfer of hydrogen in relation to hydrogen production in the dark fermentation process as a function of agitation conditions and digestate viscosity. Experiments were carried out in a baffled mechanically-stirred reactor equipped with a dualstage impeller using five levels of viscosity. Biohydrogen production was studied using glucose as substrate under controlled $\mathrm{pH}$. Three experimental techniques, namely local conductimetry, chemical decolorization and Planar Laser Induced Fluorescence were used to measure mixing time $t_{m}$ and describe the flow pattern. The effects of inter-impeller clearance and tracer injection position were also studied. Then, $\left(k_{L} a\right)_{H_{2}}$ was deduced from dynamic deaeration/aeration experiments. Experimental results showed that biohydrogen production presented a maximum in the transitional flow regime ( $R e$ about 200), and fell under turbulent flow $(R e>1000)$. Similarly, the evolution of $\left(k_{L} a\right)_{H_{2}}$ was better described by $R e$ than by the volumetric power input, contrary to literature data. Finally, the Damköhler number showed that hydrogen production was limited by liquid-to-gas mass transfer in the laminar regime and that maximum reaction rate could be reached only due to dissolved $\mathrm{H}_{2}$ supersaturation in the liquid phase. Conversely, the steep decrease of $\mathrm{H}_{2}$ production under turbulence conditions could be attributed neither to mass transfer, nor to mixing conditions, highlighting a probable negative interaction between turbulent eddies and biomass aggregates. Regarding $k_{L} a \cdot t_{m}$, the transitional flow region also approached ideal mixing,
\end{abstract}


which strengthened the conclusion that $\mathrm{H}_{2}$ production was optimized in the transition region in the dark fermentation process.

Keywords: Dark fermentation; Hydrodynamics; Mixing time; Liquid-to-gas mass transfer; Biohydrogen.

\section{Highlights:}

- Liquid-to-gas mass transfer and mixing were investigated in dark fermentation conditions.

- Mixing time, mass transfer coefficient and biological hydrogen production data were compared.

- In laminar flow, biohydrogen production was probably limited by mixing/mass transfer.

- Turbulence avoided mass transfer limitation but impaired, however, hydrogen production. 


\section{Introduction}

In the current world, primary energy supply and electricity generation are predominantly based on fossil fuels (oil, natural gas and coal), leading to carbon dioxide emissions responsible of environmental damage and climate change on a larger-scale [1]. The present situation is unsustainable and requires the development of renewable energy sources which does not involve critical pollutant emissions for environmental, economic, social, and health sustainability. In this context, hydrogen is considered, since the last decade, as an attractive solution because it can be used as an energy carrier without $\mathrm{CO}_{2}$ emission. Various methods are available for hydrogen production from biomass, which corresponds to secondgeneration biofuels, such as pyrolysis/gasification, thermal, photochemical, photoelectrochemical, photofermentation and dark fermentation (acidogenic fermentation) processes. The latter is an anaerobic process where biohydrogen, carbon dioxide and soluble metabolites are produced during the breakdown of organic compounds by the microorganisms. The soluble metabolites resulting from this process include volatile fatty acids (VFAs), i.e. acetic acid, butyric acid, propionic acid, and other products such as lactic acid and solvents [2]. This process presents the advantages to be environment friendly and sustainable because it uses renewable resources, as it can consume various organic wastes as substrates, including agricultural and food residues [3]. Despite genuine progress concerning the process development, industrial applications remain scarce, in particular because of the poor knowledge concerning the effects of the abiotic parameters on the process. Indeed, while recent research mainly addressed the effects of biotic and conventional abiotic parameters, such as $\mathrm{pH}[\mathbf{4 , 5}]$, temperature [6], substrate selection [2], byproducts concentration [7], and biohydrogen partial pressure [8-10], research should focus on the optimization of both families of factors now.

In this context, mixing is usually considered as a key parameter for the scale-up of chemical and biochemical reactors; the culture medium must be homogenized to achieve a uniform distribution of nutrients and prevent $\mathrm{pH}$ gradients in the liquid phase for enhancing bacterial growth and activity. Contrary to conventional anaerobic digestion, dark fermentation necessitates $\mathrm{pH}$ control because of VFAs accumulation during the reaction that leads to $\mathrm{pH}$ decrease and, finally, to the inhibition of the 
biohydrogen production [11]. Mixing must also ensure biogas desorption by enhancing liquid-to-gas mass transfer, so as to prevent process inhibition since a decrease in $\mathrm{pH}$ due to dissolved $\mathrm{CO}_{2}$ can switch metabolic pathway of acidogenic fermentation [12], or because dissolved biohydrogen can reach supersaturation levels that may inhibit the activity of hydrogen producing bacteria [13]. Moreover, even if high agitation speed levels lead to rapid homogenization and to more efficient heat and liquid-to-gas mass transfer, the appropriate rotation speed must be applied to prevent too high hydromechanical shear stress applied to the bacterial community under turbulent conditions which could inhibit biohydrogen production by metabolic switches, or even promote microbial lethality [14]. Finally, an optimization of these parameters is needed to establish an environment leading to enhanced biohydrogen production potential and yield. However, operating costs need also to be taken into account for the sustainability of the process, as economic sustainability needs very low power input, usually lower than $5-10 \mathrm{~W} / \mathrm{m}^{3}$ [15]. This strongly differs from the most common conditions of mixing investigated in the literature for stirred tanks. Besides, the variability in organic loading composition may lead to a wide range of digestate viscosity that may impair $\mathrm{pH}$ control and reaction yield, as pointed out by Ruggeri and Tommasi [16]. The robustness of mixing process in the dark fermentation process thus deserves further investigation.

To evaluate mixing efficiency under dark fermentation operating conditions, the mixing time and the volumetric mass transfer coefficient of hydrogen $\left(k_{L} a\right)_{H_{2}}$ can be used for scale-up purpose. The direct measurement of $\left(k_{L} a\right)_{H_{2}}$ was never reported for the characterization of liquid-to-gas mass transfer of the acidogenic fermentation process due to the lack of sensors for measuring dissolved hydrogen concentrations in culture media. For assessing the influence of agitation on mass transfer in the conditions of dark fermentation, Trad et al. [17] studied the respective effects of 9 impellers design and 3 liquid media at $25^{\circ} \mathrm{C}$ (pure water, mineral solution and digestate) on the $\left(k_{L} a\right)_{H_{2}}$ using the conventional oxygen mass transfer measurements based on the dynamic gassing-in/gassing-out method; the $\left(k_{L} a\right)_{H_{2}}$ was deduced from the experimental value of $\left(k_{L} a\right)_{O_{2}}$ with the Higbie penetration model. Macromixing time $t_{m}$ is another key parameter currently used to evaluate the mixing performance of impellers and the effect 
of hydrodynamics in stirred tanks. It is defined as the time required for achieving a certain degree of homogeneity, using a passive tracer injected in the tank [18]. Many techniques for measuring $t_{m}$ have been developed with various degrees of success in the past [19]. Recently, Ascanio [20] reviewed all the different experimental techniques used for 50 years to measure $t_{m}$ and it appears that the different methods can be divided in two groups: intrusive and non-intrusive techniques. The intrusive methods are based either on local measurements which estimate $t_{m}$ at a given location, while non-intrusive methods usually imply global measurements which deduce $t_{m}$ values from the analysis of a large area/volume with the capability to quantify and identify dead zones. The non-intrusive techniques have the advantage to avoid disturbing the flow field, contrary to the intrusive techniques where the flow field is modified by the presence of the probes. Despite the number of techniques to estimate the mixing time, there is no general accepted method because each of them exhibits limitations, as pointed out in Table $\mathbf{1}$.

Therefore, the objective of the present work is to understand the influence of the mass transfer and mixing properties of culture media on biohydrogen production in the dark fermentation process using cold flow experiments mimicking the conditions of cultures already carried out using glucose as the substrate [14]. Experiments were conducted in a mechanically stirred reactor equipped with a dual-impeller device operated under different flow regimes, so that laminar, transitional and turbulent flow conditions could be investigated. For this purpose, five levels of viscosity $\left(9.0 \cdot 10^{-4}\right.$ to $\left.6.1 \cdot 10^{-2} \mathrm{~Pa} . \mathrm{s}\right)$ and three levels of rotation speed $(40,120,200 \mathrm{rpm})$ were studied. Liquid-phase mixing times were deduced from local conductimetric, chemical decolorization and Planar Laser Induced Fluorescence (PLIF) techniques; $t_{m}$ values obtained with these different methods were critically discussed and compared. Experiments also investigated two inter-impeller clearances and several injection position in relation to the injection of the alkaline solution for $\mathrm{pH}$ control during the biological process to assess their effects on mixing time. The volumetric mass transfer of hydrogen gas was also estimated using the dynamic gassing-in/gassing-out method. Finally, the aim is to define the optimum conditions to maximize hydrogen yield and production 
potential which prevent liquid-to-gas mass transfer limitation and ensure good mixing for the scale-up of the dark fermentation process.

\section{Materials and methods}

\subsection{Stirred reactor geometry and design}

The reference configuration set-up is displayed schematically in Fig. Ia. The experiments were conducted in a fully-baffled 2-L bioreactor which consisted of a transparent borosilicate glass cylindrical vessel with an open top and a hemispherical bottom. The diameter of the baffled flat-bottomed reactor (four baffles, width $T / 10$ ) was $T=130 \mathrm{~mm}$ and the clear liquid height was $H_{L}=160 \mathrm{~mm}$. This mechanically stirred vessel was equipped with a dual-stage impeller device consisting of two six-blade Rushton turbines of $55 \mathrm{~mm}$ diameter $(D)$ and $10 \mathrm{~mm}$ height $(h)$, mounted on an aluminium shaft of $10 \mathrm{~mm}$ diameter $\left(d_{S}\right)$. The off-bottom clearance was $C_{1}=25 \mathrm{~mm}$, the inter-impeller clearance $C_{2}=85 \mathrm{~mm}$, and the distance between the top impeller and the free surface $C_{3}=50 \mathrm{~mm}$. The shaft was immersed under 10 $\mathrm{mm}\left(C_{S}\right)$ of the bottom of the vessel and driven clockwise by a variable speed electric motor (Eurostar 60 Control, IKA ${ }^{\circledR}$-Werke $G m b H$, Germany). The injection point position of passive tracers in the reference configuration was at the surface of the liquid: so, $P_{I}=H_{L}=160 \mathrm{~mm}$, corresponding to the injection of the alkaline solution for $\mathrm{pH}$ control in the dark fermentation process.

Alternative configurations including another inter-impeller clearance $C_{2 \prime}=115 \mathrm{~mm}$ and another injection point position $P_{I^{\prime}}=110 \mathrm{~mm}$ were studied to define the respective effects of these parameters on the value of the mixing time. For this purpose, three configurations were studied:

$\checkmark$ Alternative configuration 1: $C_{2}=85 \mathrm{~mm}, P_{I^{\prime}}=110 \mathrm{~mm}($ Fig. $\mathbf{l b})$;

$\checkmark$ Alternative configuration 2: $C_{2 \prime}=115 \mathrm{~mm}, P_{I}=160 \mathrm{~mm}$ (Fig. 1c);

$\checkmark$ Alternative configuration 3: $C_{2^{\prime}}=115 \mathrm{~mm}, P_{I^{\prime}}=110 \mathrm{~mm}($ Fig. 1 d $)$. 


\subsection{Operating conditions}

The viscosity of the liquid phase at $35^{\circ} \mathrm{C}$ was adjusted from $9.0 \cdot 10^{-4}$ to $6.1 \cdot 10^{-2}$ Pa.s by adding viscous solutions of water soluble polyalkylene glycol Emkarox ${ }^{\mathrm{TM}} \mathrm{HV} 45$ to distilled water (Table 2). The rheological properties of these Newtonian solutions were measured using a stress-controlled rheometer AR-G2 (TA Instruments, USA) equipped with a double-walled Couette geometry fitted with a cell temperature control device set to $35^{\circ} \mathrm{C}$, and the Advantage ${ }^{\circledR}$ software was used for instrument control and data collection. Each solution was driven at three values of rotation speed: $0.67,2.00$ and $3.33 \mathrm{~s}^{-1}$; corresponding to 40, 120 and $200 \mathrm{rpm}$, respectively. In total, 15 viscosity/speed conditions were studied in triplicate for each configuration by each method in order to minimize experimental error and ensure reproducibility. As the viscosity of the solutions varied significantly with the temperature, the experiments were conducted at a constant controlled temperature of $35^{\circ} \mathrm{C}$. Before each experiment, the solutions were maintained $30 \mathrm{~min}$ at the corresponding rotation speed and temperature to prevent the presence of air bubbles and ensure established flow and temperature conditions.

In a recent work, Chezeau and Vial [14] demonstrated that the combined influence of viscosity and rotation speed in a stirred tank bioreactor in which dark fermentation is operated can be optimized as a function of the purely hydrodynamic dimensionless Reynolds number $R e$. Number. $R e$ for a conventional stirred tank is defined as:

$$
R e=\frac{\rho N D^{2}}{\mu}
$$

where $\rho$ is the density of the fluid, $N$ the impeller rotation speed, $D$ the diameter of the impellers used and $\mu$ the fluid dynamic viscosity. $R e$ is a dimensionless parameter which represents the ratio of the inertial to the viscous forces. The transition from laminar to transitional flow occurs when $R e$ is less than 100 , and the tank is highly turbulent by $R e$ superior to 1000 [21]. The transition between laminar and turbulent flows is known as laminar-turbulent transition and even today, transitional flows are not fully understood. In this work, $R e$ varied from 30 to $1.1 \cdot 10^{4}$ and this range was used to study mixing time and liquid-to-gas 
mass transfer, as it corresponds to a volumetric power input between 1 and $100 \mathrm{~W} / \mathrm{m}^{3}$, which includes the typical values $5-10 \mathrm{~W} / \mathrm{m}^{3}$.

\subsection{Measurements of the mixing time}

Mixing time measurements were measured in non-reactive and unaerated flow using conductimetric, chemical decolorization and PLIF methods; experiments were conducted using a similar procedure for comparison purpose: this includes the same volume $(2 \mathrm{~mL})$ for the injection of the passive tracer, and an injection performed on a very short time to mimic an instantaneous tracer input function (Dirac-type $\delta$ ). For each method, the value of mixing time was estimated for a 95\% homogeneity.

\subsubsection{Conductimetric method}

The conductimetric method or "local conductivity tracer technique" based on pulse injection was applied to estimate the mixing time $t_{m}$. A conductivity probe CDC 241-9 (cell constant $1.012 \mathrm{~cm}^{-1}$ ) supplied with a digital conductivity-meter CDM210 (Radiometer Analytical, France) was utilized for tracer detection at $1 \mathrm{~Hz}$ frequency using a data recorder (USB DAQ-1901, Adlink Technology, USA). Sodium chloride was selected as the tracer because of its non-reactive nature and its negligible effect on the physical properties of the fluid (density, viscosity), with the exception of the conductivity of distilled water. The injection of the $\mathrm{NaCl}$ solution $(1.0 \mathrm{M})$ was carried out manually with a syringe of $6.0 \mathrm{~mL}$ at the same positions reported in the previous sections. The detection of the signal was operated by the conductivity probe localized at the position $C_{2}=85 \mathrm{~mm}$; this probe allowed the continuous measurement of the conductivity and, after calibration, the estimation to the local salt concentration $C(t)$ in the solution. The proportionality between salt concentration and conductivity could be assumed in diluted solution, so that $t_{m}$ could be deduced.

\subsubsection{Chemical decolorization method}

The chemical decolorization method or "acid-base decolorization technique" was applied to estimate the mixing time and evolution in the tank reactor. This method based on image analysis consists in 
recording the color change of the solution depending on pH. A digital camera (Canon EOS 70D, Canon, Japan) equipped with a camera lens EF 24-105 mm f/3.5-5.6 IS STM (Canon, Japan) was utilized to record video sequences at $30 \mathrm{fps}\left(1920 \times 1080\right.$ pixels $\left.^{2}\right)$. The stirred tank was immersed in a square vessel $\left(40 \times 40 \mathrm{~cm}^{2}\right)$ filled with distilled water to reduce optical distortion, and was illuminated by a LED panel (LEDW-BL-300/220-LLUB-Q-1R24, Phlox Corp., France) placed on the opposite side using an ombroscopy technique. This enables to have white light with a high uniformity of the light intensity $( \pm 5 \%)$, a color temperature of $5000 \mathrm{~K}$, and to operate in the backlight mode and quasi-isothermally to avoid heating tank. Bromocresol purple was used as the $\mathrm{pH}$ indicator and was added in the tank at a concentration of $4 \cdot 10^{-2} \mathrm{~g} / \mathrm{L}$. The tracer is yellow when the $\mathrm{pH}$ solution is below 5.2 (acid color) and purple when the $\mathrm{pH}$ solution is above 6.8 (alkaline color). Mixing time was measured for an alkaline to acid reaction i.e. a color evolution from purple to yellow; this allows an easier detection of the unmixed zones. Before $t_{m}$ measurements, the solutions were set to the purple color by adding $1 \mathrm{~mL}$ of an aqueous solution of $\mathrm{NaOH}(5.0 \mathrm{M})$ and the measurements started when adding $2 \mathrm{~mL}$ of an aqueous solution of $\mathrm{HCl}(2.5 \mathrm{M})$ as the tracer with a syringe of $6.0 \mathrm{~mL}$ at the positions already reported in the previous sections.

The resulting images were analyzed with a Matlab ${ }^{\circledR}$ (The MathWorks, USA) code. This consisted in creating a masking wall and internals with PhotoFiltre freeware, and the mixing time was deduced by following the evolution of the HSV (Hue Saturation Value) value of each pixel in the pictures in the working zone.

\subsubsection{Planar Laser Induced Fluorescence method (PLIF)}

The mixing time and evolution in the tank reactor was also studied using the PLIF method. This technique based on image analysis allows determining the instantaneous concentration field of a tracer in the plane lighted by a laser. The experimental setup included a monochrome camera (UI-3370CP-M-GL, IDS GmbH, Germany) placed perpendicularly to the laser sheet, equipped with a camera lens 23-FM-25SP (Tamron, Japan) and with a high pass optical filter $(550 \mathrm{~nm}$ to remove the light scattered by laser reflections) to record video sequences at $10 \mathrm{fps}\left(2048 \times 2048\right.$ pixels $\left.{ }^{2}\right)$, and a Raypower Nd:Yag laser 
(Dantec Dynamic SA, Denmark) emitting light at $532 \mathrm{~nm}$ wavelength and operated in the continuous mode which illumined a vertical plane parallel to the shaft of the impellers. As in the chemical decolorization technique, the stirred tank was immersed in a squared vessel filled with distilled water to reduce optical distortion. A Rhodamine B (peak absorption $555 \mathrm{~nm}$ and peak emission $580 \mathrm{~nm}$ ) solution was used as the passive tracer and was injected with a syringe of $6.0 \mathrm{~mL}$ to achieve a final passive tracer concentration of $5 \mu \mathrm{g} / \mathrm{L}$ in the tank reactor.

A method similar to that of Busciglio et al. [22] was applied with a Matlab ${ }^{\circledast}$ (The MathWorks, USA) code for image analysis. This consisted in masking walls and internals with the PhotoFiltre freeware, separating the background from a set of images before tracer injection, and defining the grey level when mixing was achieved from a set of images at the end of the experiments; thus, a dimensionless index between 0 and 100 could be defined for each pixel. Mixing was finally assessed through the monitoring of the coefficient of variation of this index in each image over time, which was normalized to account for image noise before injection, once homogenization was achieved. Finally, the mixing time was defined as the time necessary to achieve a value lower than 0.05 .

\subsection{Measurements of the volumetric mass transfer coefficient}

$\left(k_{L} a\right)_{O_{2}}$ was measured using dynamic deaeration/aeration experiments based on air and nitrogen gas supply alternatively. The experiments were conducted without biochemical reactions in the same fluids and under the same agitation conditions already reported at a constant gas flow rate $(5.0 \mathrm{~L} / \mathrm{h}$, i.e. 0.042 $\mathrm{vvm}$ ), corresponding to the highest levels of biogas production rate reported in the biohydrogen production process. The volumetric mass transfer coefficient of hydrogen $\left(k_{L} a\right)_{H_{2}}$ was deduced from experimental $\left(k_{L} a\right)_{O_{2}}$ using the Higbie penetration model, which expresses the mass transfer coefficient in the liquid film $k_{L}$ as a function of the molecular diffusivity $\left(D_{i}\right)$ of species $i$ in water:

$$
\left(k_{L}\right)_{H_{2}}=\left(k_{L}\right)_{O_{2}} \cdot \sqrt{\frac{D_{H_{2}}}{D_{O_{2}}}}
$$


At $35^{\circ} \mathrm{C}, D_{\mathrm{H}_{2}}=6.3 \cdot 10^{-9} \mathrm{~m}^{2} / \mathrm{s}$ while $D_{\mathrm{O}_{2}}=3.2 \cdot 10^{-9} \mathrm{~m}^{2} / \mathrm{s}$, leading to a correction factor about 1.4 . Thus, at constant interfacial area $a,\left(k_{L} a\right)_{H_{2}, 35^{\circ} \mathrm{C}}$ is defined as:

$$
\left(k_{L} a\right)_{H_{2}, 35^{\circ} \mathrm{C}}=1.4 \cdot\left(k_{L} a\right)_{O_{2}, 35^{\circ} \mathrm{C}}
$$

Volumetric mass transfer measurements were conducted using a dissolved oxygen micro-sensor (OX500-15019, Unisense A/S, Denmark). At the beginning of every experiment, the microprobe was calibrated and then mounted on a micromanipulator system, so that it was immersed at $10 \mathrm{~mm}$ from the vessel wall and $50 \mathrm{~mm}$ below the free surface. It was connected to an oxymeter controlled by the SensorTrace Pro ${ }^{\circledR}$ software, which allowed to convert the electric signal into dissolved oxygen concentration. Gas was introduced through a stainless steel sparger (hole diameter: $2 \mu \mathrm{m}$ ) located under the lower impeller at mid-height of the off-bottom clearance, leading to tiny bubbles.

In this work, gas-liquid mass transfer was slow with cycles of gassing-in/gassing-out between $30 \mathrm{~min}$ and $4 \mathrm{~h}$ long. Consequently, the response time of the microprobe (15 s) was neglected, the liquid phase was considered as perfectly mixed and no first-order correction of the microprobe signal was necessary. Finally, the volumetric mass transfer coefficient could be deduced from Eq. (4) in gassing-in experiments and from Eq. (5) in gassing-out experiments, respectively.

$$
\begin{aligned}
& \ln \left(\frac{C_{\max }-C_{\min }}{C_{\max }-C_{(t)}}\right)=k_{L} a \cdot t \\
& \ln \left(\frac{C_{\max }-C_{\min }}{C_{(t)}-C_{\min }}\right)=k_{L} a \cdot t
\end{aligned}
$$

where $C_{\min }$ and $C_{\max }$ are the minimum and maximum concentrations of dissolved oxygen, respectively, while $C_{(t)}$ is the dissolved oxygen concentration measured at time $t$.

The bubble size distribution was studied to ensure that the interfacial area $a$ was not affected by viscosity. For this purpose, pictures were captured at 2 min intervals by a digital camera (Canon EOS 70D, Canon, Japan) provided with a camera lens EF 24-105 mm f/3.5-5.6 IS STM (Canon, Japan). The analysis of the pictures was carried out using ImageJ $^{\circledR}$ (National Institutes of Health, USA); graduation 
was put on the vessel wall for calibration, and the resulting bubble size distribution could be usually described using a normal distribution.

Nevertheless, it must be pointed that gas injection does not accurately represent actual in situ nucleation of bubbles in the dark fermentation process. Indeed, even if no studies reported the description of bubble nucleation in acidogenic fermentation, it might be hypothesized that the nucleation process can occur everywhere in the culture medium and release bubbles at various frequencies, which is not the case when gas is dispersed. However, the $\left(k_{L} a\right)_{H_{2}}$ estimation provides relevant information to better understand the hydrogen supersaturation in the liquid phase.

\section{Results and discussion}

In this section, the experimental results of $t_{m}$ and $\left(k_{L} a\right)_{H_{2}}$ are first discussed and analyzed as a function of rotation speed and fluid viscosity. Then, a relationship between hydrogen production, liquidto-gas mass transfer and mixing is developed to improve scale-up methodology of the dark fermentation process.

3.1. Mixing time analysis and influence of the measuring method

Experimental data on $95 \%$ mixing time derived from conductimetric, chemical decolorization and PLIF experiments according to the reference configuration are reported in Table 3. The comparison of the mixing time data obtained with the three methods highlights that the results are very close. However, some discrepancies can be observed, especially when the dimensionless Reynolds number $R e$ is between 150 and 500 , i.e. under transitional flow conditions. In this case, the resulting $t_{m}$ values with the conductimetric technique are higher than those obtained with the chemical decolorization and PLIF techniques. From Table 1, these discrepancies can be explained because the conductimetric method is local and intrusive, which may therefore disturb the flow close to the probe. To prevent the disturbing effect of the probe on the flow, Giona et al. [23] reported the use of probes attached to the baffle system or on the shaft. Moreover, $t_{m}$ values recorded with the conductimetric method are highly dependent of the probe position especially in the laminar regime, while Bouwmans et al. [24] found that in the turbulent 
regime, the probe position does not exhibit a significant effect on the mixing time measurement. Contrary to the local conductimetric technique, chemical decolorization and PLIF techniques are supplemented with digital image processing, which results in more accurate measurement methods allowing to discriminate the segregated and well-mixed regions during mixing [25-27]. It may be advocated that chemical decolorization is based only on a front image, so that regions of the reactor which are not mixed might not been seen and mixing time is underestimated; but this issue is circumvented by PLIF in which a planar region is lighted, so that good mixing conditions close to the wall cannot hide mixing conditions in the core region of the tank. According to Fig. 2, the influence of flow regime and the occurrence of segregated and well-mixed regions can be observed under laminar (Figs. $2 \boldsymbol{a}$ and $2 \boldsymbol{b}$ ) and turbulent (Figs. $2 \boldsymbol{c}$ and $2 \boldsymbol{d}$ ) regimes with the chemical decolorization and PLIF techniques, respectively. Thus, the use of these methods is particularly beneficial to discriminate the flow patterns even if they have the disadvantage to be expensive, particularly the PLIF method that requires the use of both camera and laser [20].

As pointed out by Chezeau and Vial [14], hydrogen production data as a function of rotation speed and viscosity could be expressed on a master curve as a function of $R e$. The same methodology was applied to $t_{m}$ in Fig. 3 for the three methods. This plot highlights that whatever the fluid viscosity, the same conclusion could be made and that $t_{m} v s . R e$ also displayed a master curve of mixing time. As it can be seen, $t_{m}$ decreases as $R e$ increases in the laminar flow region $(R e<100)$ and in the fully turbulent regime $(R e>1000)$ when $R e$ varies from 30 to $1.1 \cdot 10^{4}$. Using the conductimetric technique, it is noteworthy that the evolution of $t_{m} v s$. Re can be described by two straight lines in the semilog plot of Fig. $\mathbf{3 a}$; the first one where $t_{m}$ falls steeply corresponds to the laminar-flow regime up to $R e=300$, while the second one where $t_{m}$ slowly decreases is characteristic of the transitional-turbulent regime $(\operatorname{Re}>300)$. Conversely, only the first straight line can be observed in Figs. $3 \boldsymbol{b}$ and $3 \boldsymbol{c}$ using chemical decolorization and PLIF, respectively. In the transition region $(100<R e<1000), t_{m}$ exhibits a local minimum around $R e=450$, followed by a local maximum below $R e=1000$. This illustrates why $t_{m}$ values differ in Table 3 in the transitional flow regime in which the local intrusive method deviates from the values of both $2 \mathrm{D} / 3 \mathrm{D}$ 
visualization techniques. Conversely, it must be pointed out from Table 3 that below a value of $10 \mathrm{~s}$, the decolorization technique may underestimate mixing time when poorly mixed areas are in the core region of the tank, whereas $t_{m}$ approaches the response time of conductimetric probes. In this case, PLIF emerges as a good trade-off.

Finally, Table 3 and Fig. 3 display that $t_{m}$ falls from about $240 \mathrm{~s}$ when $R e=30$ to values close to 110 $\mathrm{s}$ when $R e=100$ in the laminar region, while it decreases from $18 \mathrm{~s}$ to $4 \mathrm{~s}$ when $R e$ increases from 1000 to $1.110^{4}$ in the fully turbulent region. In the dark fermentation process, these values measure the time necessary for concentration homogenization when alkaline solution is added to maintain constant $\mathrm{pH}$. It is, therefore, a characteristic time of the process that can be compared to the characteristic times of mass transfer and biohydrogen production, respectively.

\subsection{Influence of injection position and impeller clearance on mixing time}

To assess the influence of viscosity and rotation speed on mixing, the influence of the injection position and the impeller clearance were investigated. As shown in Fig. 1, reference design and configuration 2 correspond to an increase of the inter-impeller clearance with the same injection point, while configurations 1 and 3 correspond to the previous ones with a change of the injection position, respectively.

Fig. 4 displays pictures of the evolution of mixing using the decolorization technique for both impeller designs and injection positions when $R e=33$. This figure highlights the strong differences in mixing time between the configurations. First, considering the influence of injection position, the comparison between $t_{m}$ values is plotted in Fig. 5a. This displays a linear trend with a slope of $1.12 \pm 0.03\left(R^{2}>0.99\right)$ between configuration 1 and the reference design, and of $1.08 \pm 0.03\left(R^{2}>0.99\right)$ between configuration 3 and configuration 2, respectively. In detail, this difference mainly results from the laminar flow conditions; i.e. when $R e<100$ : this means that the same plot for $R e>100$ between configuration 1 and the reference design also provides a linear slope with a slope around $1.06 \pm 0.04\left(R^{2}>0.98\right)$, which highlights that the differences in mixing time are reduced in transitional and turbulent flows. The same 
conclusions can be drawn with the three measuring techniques, even though the same discrepancy between the conductimetric technique on the one hand, and the two others on the other hand, already reported in the transition region in section $\mathbf{3 . 1}$ can also be observed. The conclusion is that faster mixing is always observed when injection is located at the top of the tank than at mid-height in the reactor.

In Fig. 4, these differences can be explained by the well-known overall cascade structure that prevails in multiple-turbine agitated tanks [28]. When the acidic solution was injected in the top region (Fig. 4a), this region was rapidly mixed, and the tracer reached also rapidly the inter-impeller zone and, finally, the bottom of the tank. Conversely, when this solution was injected in the discharge of the lower impeller (Fig. 4b), the spherical bottom of the reactor, which distorted the streamlines in the lower part of the tank, always promoted fast mixing in the bottom region, but delayed mixing in the upper part of the tank which behaved as a stagnant zone between $150 \mathrm{~s}$ and $210 \mathrm{~s}$. Changing the position of the upper disk turbine did not reduce mixing time in this case, as the faster mixing in the upward region of the tank was counterbalanced by slower mixing rate in the inter-impeller region (Fig. $4 \mathbf{c}$ ), which finally lead to higher $t_{m}$ values. These results highlight that mixing clearly depends on the injection point in the laminar and the transitional flow regimes, but they also highlight the key role of a dual-impeller design that avoids biomass settling and prevents segregation in the upward region of the tank at the same time. With a single impeller, Collignon [29] reported that $t_{m}$ values were 1.2 to 2.5 times higher when the injection was close to the free surface than when it was operated close to the wall of the tank at the level of the impeller discharge. Even though the free surface remains a region very difficult to homogenize in this work from Fig. 4, the upper turbine in the dual-stage geometry enhances the circulation between the top and the middle of the tank, which makes possible the addition of an alkaline solution for $\mathrm{pH}$ control in dark fermentation close to the free surface with a $\mathrm{pH}$ probe in the inter-impeller region to prevent $\mathrm{pH}$ gradients. A similar conclusion had been drawn by Trad et al. [30], but in an unbaffled vessel in which the flow field thus significantly differs. Finally, these results highlight that the injection of the alkaline solution for $\mathrm{pH}$ 
control in the dark fermentation experiments is more appropriate at the top of the tank in laminar and transitional flow conditions to achieve faster mixing, and prevent segregated regions and $\mathrm{pH}$ gradients.

Regarding the effect of the impeller position, Fig. $5 \boldsymbol{b}$ exhibits the same linear trends between configurations when the position of the upper impeller is modified at fixed injection point. This shows a slope around $1.33 \pm 0.03\left(R^{2}>0.99\right)$ between configuration 2 and the reference design, and $1.28 \pm 0.04$ $\left(R^{2}>0.99\right)$ between configuration 3 and configuration 1 , respectively. The effect of impeller position is more influential than injection position, as pointed out by the values of the slopes. As already stated, major differences between the two inter-impeller configurations resulted from the mixing efficiency in inter-impeller region. Effectively, when the clearance was set to $C_{2 \prime}=115 \mathrm{~mm}$, the circulation between both impellers was too weak to induce an efficient mixing between the top and the bottom of the tank, whatever the position of the injection point. For example, mixing in the inter-impeller region of the tank was complete after $210 \mathrm{~s}$ with $C_{2}=85 \mathrm{~mm}(\boldsymbol{F i g} . \mathbf{4 a})$, while $300 \mathrm{~s}$ were necessary with $C_{2 \prime}=115 \mathrm{~mm}(\boldsymbol{F i g}$. $4 c$ ), This means that mixing is enhanced when $C_{2} / D$ is close to 1.5 . As a conclusion, accounting for these results, the reference configuration with $C_{2}=85 \mathrm{~mm}$ and $P_{i}=160 \mathrm{~mm}$ emerges as a good compromise to obtain faster mixing under laminar to turbulent flow conditions.

In order to analyze mixing effectiveness more deeply, the dimensionless mixing time $N t_{m}$ could be plotted as a function of $R e$. This plot is presented in Fig. $\mathbf{6} \boldsymbol{a}$ and displays that, as expected, $N t_{m}$ is constant under turbulent flow conditions in a baffled vessel because it is only a function of the impeller-totank diameter ratio $D / T$ and is expressed as follows for a single Ruston turbine [31]:

$$
N \cdot t_{m}=5.3 \cdot N_{p}^{-1 / 3} \cdot\left(\frac{D}{T}\right)^{-2}
$$

where $N_{p}$ is the power number defined as:

$$
N_{p}=\frac{P}{\rho N^{3} D^{5}}
$$

where $P$ is power input into the reactor due to mechanical stirring, and $\rho$ is fluid density. This gives $N t_{m}=15$. Fig. $6 \boldsymbol{a}$ displays $N t_{m}$ about $12 \pm 3$, which is rather close to the expected value, even though a 
dual-stage impeller with a $H / D=1.23$ is used, achieving more rapidly homogeneity than a single Rushton turbine. But Fig. $\boldsymbol{\sigma} \boldsymbol{a}$ also shows that $N t_{m} v s . R e$ does not follow a simple trend when $R e<300$ and provides values that depend on the viscosity. This effect has been accounted by Grenville and Nienow [32] in transitional flow who pointed out that for the same geometry, mixing time can be derived from the following expression:

$$
N_{p}^{1 / 3} \cdot R e \cdot \sqrt{F o}=184
$$

where $F_{O}$ is a mixing Fourier number:

$$
F o=\frac{\mu t_{m}}{\rho T^{2}}
$$

Eq. (8) and Eq. (9) can be generalized as follows:

$$
N_{p}^{1 / 3} \cdot R e \cdot F o^{m}=c
$$

where $c$ is a constant and $m$ an exponent that depend on the flow regime, with $m=1 / 2(c=184)$ and $m=$ $1(c=5.2)$ under laminar and turbulent flow conditions, respectively [28]. Using torque measurements and $N_{p}$ data from Chezeau and Vial [14], the generalized plot of Eq. (10) is displayed in Fig. $\boldsymbol{6} \boldsymbol{b}$ for the four configurations. As mentioned by Grenville and Nienow [32], the transition emerges when $1 / F o$ is about 1000 for a single-stage impeller and is observed at smaller $1 / F o$ values in multi-stage impeller designs [28]. This conclusion agrees with $\boldsymbol{F i g}$. $\boldsymbol{6} \boldsymbol{b}$, with two straight lines exhibiting slopes that comply with the 1/2 and 1 values. The constant $c$ in the transition region in Fig. $\boldsymbol{\sigma} \boldsymbol{b}$ is, however, close to 100, which differs significantly from $c=184$, but this can probably be explained by the use of a dual-impeller design. All the configurations exhibit the same trends, so that a master curve can be deduced in $\boldsymbol{F i g}$. $\boldsymbol{\sigma} \boldsymbol{b}$ for each configuration, while $N t_{m}$ becomes always constant when turbulent flow is achieved ( $m=1$, and constant $N_{p}$ ), with a higher value $N t_{m}=23 \pm 5$ when the inter-impeller clearance is increased (configurations 2 and 3). Finally, these results highlight that mixing time data agree with the literature and that either experimental $t_{m}$ values or Eq. (10) can be used for further comparison of the characteristic times of macromixing, mass transfer, and biochemical reaction. Besides, to avoid $\mathrm{pH}$ gradients, $t_{m}$ values in Table 
3 and Fig. 3 show that laminar flow conditions should be avoided, and that transitional flow constitutes a trade-off for energy savings.

\subsection{Analysis of liquid-to-gas mass transfer}

First, bubble size was investigated as a function of rotation speed and fluid viscosity. Due to the effectiveness of the sparger, the influence of rotation speed was negligible in comparison to viscosity. Bubbles were always spherical and homogeneously dispersed, which may fit the dispersion encountered in dark fermentation. Bubble size distributions were nearly Gaussian and fitted using a normal distribution. Fig. 7 displays the fitted curves: the average bubble size increased as a function of the viscosity of the liquid phase. Indeed, the average diameter of the bubbles was $0.39,0.42,0.44,0.47$ and $0.55 \mathrm{~mm}$ for the medium viscosity of $9.0 \cdot 10^{-4}, 2.5 \cdot 10^{-3}, 2.3 \cdot 10^{-2}, 3.7 \cdot 10^{-2}$, and $6.1 \cdot 10^{-2} \mathrm{~Pa} . \mathrm{s}$, respectively. These results indicate that viscosity can impact the bubble size distribution and change the interfacial area $a$, even though surface tension was not significantly different, and may reduce $k_{L} a$ when it increases.

The $\left(k_{L} a\right)_{H_{2}}$ estimation was derived from the experimental values of $\left(k_{L} a\right)_{O_{2}}$ using the Higbie penetration model, as reported in the section 2.4. Fig. $8 \boldsymbol{a}$ displays the evolution of $\left(k_{L} a\right)_{H_{2}}$ as function of $R e$. As mixing time, but also hydrogen production potential, $\left(k_{L} a\right)_{H_{2}}$ shows a continuous evolution $v s$. Re when both viscosity and rotation speed are changed, which is not the case as a function of power input (Fig. $\boldsymbol{8 b}$ ). This behavior clearly differs from aerobic fermenters in which $k_{L} a$ is usually expressed as a function of $P / V$ at constant gas velocity [33], which does not account for the effect of viscosity in the present work $(\boldsymbol{F i g} . \mathbf{8 b})$. As it can be seen in Fig. $8 \boldsymbol{a}$, reported values were lower between $6.1-10.8 \cdot \mathrm{h}^{-1}$ at $R e<100$; and hydrogen mass transfer increased steeply under transitional and turbulent flows to approach a maximum value of $100 \mathrm{~h}^{-1}$ when $R e$ reached $1.1 \cdot 10^{4}$. It must be pointed out that this evolution of $\left(k_{L} a\right)_{H_{2}}$ can be explained by the fact that from transitional to turbulent flow conditions, the formation of turbulent eddies improves more efficiently the mass transfer properties of the flow. Interestingly, as reported above in section 3.1, the evolution of $\left(k_{L} a\right)_{H_{2}}$ as a function of $R e$ can be also described with two nearly linear trends in the semilog plot: the first one has a slight slope and is characteristic of the laminar- 
transitional regime, while the second one has a high slope and is characteristic of the transitional-turbulent regime. In practice, $\left(k_{L} a\right)_{H_{2}}$ varied as $R e^{0.65}$ and $R e^{0.3}$ in the laminar-transitional and the turbulent flow regimes, respectively.

From a theoretical point of view, $k_{L} a$ is a function of mixing conditions, viscosity and diffusivity, which is affected by viscosity. The Stokes-Einstein approximation is usually applied, which states that diffusivity is inversely proportional to viscosity. As $k_{L} a$ depends on $R e$ and the dimensionless Schmidt number, it becomes only the function of $N$ and $\mu$ in this work, assuming that the Stokes-Einstein equation is applied. Thus, one deduces that:

$$
k_{L} a=c N^{\alpha} \mu^{\beta}
$$

But it cannot be assumed a priori that $\alpha=-\beta$. Linear regression was therefore applied using Eq. (11) and the results showed that $\alpha=0.53, \beta=-0.61$ when $R e<1000\left(R^{2}>0.95\right)$, and $\alpha=0.32, \beta=-0.23$ when $R e>1000\left(R^{2}>0.95\right)$, which agrees roughly with the fact that $\left(k_{L} a\right)_{H_{2}}$ is only a function of $R e$ in this work.

The results from Fig. 8 can also be compared to the literature. As previously mentioned, direct measurements of $\left(k_{L} a\right)_{H_{2}}$ were never reported before for the acidogenic fermentation process, but deduced as in this work or from mass balance. Thus, the values of this work are higher than those obtained in the conditions of dark fermentation by Zhang et al. [13] and Trad et al. [17], who reported $\left(k_{L} a\right)_{H_{2}}$ between $6.8-10.2 \mathrm{~h}^{-1}$ and $3.2-6.7 \mathrm{~h}^{-1}$, respectively. This can be explained, in part because of the better mixing conditions than in Zhang et al. [13] who used magnetic stirring, as highlighted in sections 3.1 and 3.2, and because of the quality of the gas distribution which was far more homogeneous than in Trad et al. [17] due to the presence of baffles.

Finally, these results highlight that $\left(k_{L} a\right)_{H_{2}}$ is only a function of $R e$ that increases steeply in the transition and turbulent flow regimes. As expected, viscosity and agitation conditions exhibit a significant effect on $k_{L} a$. Agitation may be used to enhance mass transfer or counterbalance the effect of viscosity. It also emerges that laminar conditions lead to low $k_{L} a$, whereas gas-liquid mass transfer is strongly 
enhanced in transitional and turbulent flow regimes. A key result is also, as in section 3.2 on mixing time, that transitional flow conditions may constitute a trade-off between the need for hydrogen mass transfer conditions and energy savings in the dark fermentation process.

\subsection{Relationship between hydrogen production, liquid-to-gas mass transfer and mixing}

To investigate the relationship between hydrogen production potential, liquid-to-gas mass transfer and mixing; the dark fermentation results of Chezeau and Vial [14] obtained in the same conditions of agitation speed and viscosity have been used on the basis of the reference configuration which provided the smallest $t_{m}$ values. The main conclusions of their work is that the combined influence of agitation speed and viscosity in a stirred tank bioreactor in which acidogenic fermentation is operated must be expressed as function of the purely hydrodynamic dimensionless $R e$, and that an optimum can be found in the transitional regime. Using the Gompertz equation for analyzing the batch experiments, the cumulative hydrogen production $H(t)$ can be expressed as:

$$
H(t)=H_{\max } \cdot \exp \left\{-\exp \left[\frac{R_{\max } \cdot \exp (1)}{H_{\max }} \cdot(\lambda-t)+1\right]\right\}
$$

where $H_{\max }$ is the hydrogen production potential of the substrate in $\mathrm{L}, \lambda$ is the lag time in $\mathrm{h}$ and $R_{\max }$ the maximum hydrogen production rate in L/h. So, two characteristic reaction times can be deduced from Eq. (12): the lag time $\lambda$, and a maximum specific $\mathrm{H}_{2}$ production rate $R_{\max } / V$. These can thus be compared to the macromixing time $t_{m}$ that characterizes the ability to achieve homogeneous $\mathrm{pH}$ in the tank, and to the characteristic time of hydrogen mass transfer for desorption $1 / k_{L} a$. The results are summarized in Table

\section{4.}

First, Table 4 shows that the lag time increases when viscosity increases at constant rotation speed and becomes nearly constant when $R e$ is higher than 800 , about $10 \mathrm{~h}$, which seems to correspond to the minimum value. When $R e<800, \lambda$ is higher, but cannot be described only by the evolution of $R e$, which highlights that not only diffusivity/viscosity, but also other parameters may be involved, such as the adaptation of the microorganism to a different chemical environment with a lower water activity. Then, Fig. $9 a$ describes the effect of $R e$ on hydrogen production potential. This increases and peaks between 
$R e>100$ and $R e<200$. But under turbulent flow conditions when $R e>1000$, hydrogen production potential decreases steeply to approach the minimum value of this work when $R e$ is the highest $(R e=$ $\left.1.1 \cdot 10^{4}\right)$. As it can be seen, turbulent conditions impair the dark fermentation process, which could be explained by a damaging effect of turbulent eddies on microorganism aggregates [14].

At the same time Fig. $\mathbf{9 b}$ illustrates the experimental results of volumetric mass transfer of hydrogen and mixing time obtained with the chemical decolorization method as function of the $R e$. The dotted lines in the Fig. 9 highlight the area where maximum values of hydrogen production are reported, corresponding to transitional conditions. To compare the characteristic times of mixing, mass transfer, and reaction, two dimensionless numbers were defined: $k_{L} a \cdot t_{m}$ which directly compares the mixing and mass transfer times, and the Damköhler number $D a$ which compares the maximum hydrogen production rate $\rho_{\mathrm{H} 2} R_{\max } / V$ to the maximum mass transfer rate $k_{L} a \cdot C^{*}$, both expressed in $g_{H_{2}} /\left(L_{\text {reactor }} \cdot h\right)$ as follows:

$$
D a=\rho_{H 2} \frac{R_{\max } / V}{k_{L} a \cdot C^{*}}
$$

where $R_{\max }\left(L_{\mathrm{H}_{2}} / \mathrm{s}\right)$ is derived from Eq. (13) (data from Table 4), $V$ the volume of the liquid phase in the bioreactor, $C^{*}$ the solubility of hydrogen gas in water at $35^{\circ} \mathrm{C}$ (about $1.4 \mathrm{mg} / \mathrm{L}$ under atmospheric pressure), and $\rho_{H 2}$ the hydrogen gas density deduced from ideal gas law.

Fig. 10 shows first that $\mathrm{Da}$ lies between 4-7 in the laminar flow regime, decreases in the transitional region and turbulent flow conditions, becoming lower than 1 when $R e$ is between 1000-2000. Theoretically, $\mathrm{Da} \gg>1$ corresponds to fast reaction and kinetic limitation by mass transfer. This situation prevails when $R e<100$. In practice, because $R_{\max }$ estimation in Eq. (13) is based on measurements in the gas phase, the theoretical consequence is that $\rho_{\mathrm{H} 2} R_{\max } / V \leq k_{L} a \cdot C^{*}$, so that the maximum $D a$ value should be 1 in this work. $D a>1$ thus leads to the same conclusion as Zhang et al. [13], despite they applied a rather different approach, i.e. that the supersaturation of $\mathrm{H}_{2}$ in the liquid phase prevails and that $C^{*}$ is underestimated. When $R e>100, D a$ decreases from 5 to about 0.4 which highlights that maximum reaction and mass transfer rates become rather close, so that mass transfer limitation progressively 
vanishes at increasing $R e$. However, it must be pointed out that $R_{\max }$ increases $v s$. Re in the laminar regime, while it decreases in turbulent flows (Table 4), whereas $k_{L} a$ is always an increasing function of $R e$. The decrease in $R_{\max }$ and $H_{\max }$ when $R e>1000$ cannot therefore be attributed to a mass transfer limitation. The same conclusion can be drawn for mixing, using $k_{L} a \cdot t_{m}$ (Table 4) which lies about 0.2 in turbulent flow. As noticed by Chezeau and Vial [14], these decreases are observed when the Kolmogorov length scale approaches the size of biomass aggregates.

Table 4 also highlights that $k_{L} a \cdot t_{m}$ ranges between 0.03 and 0.4 , is maximum about 0.4 in laminar conditions, and minimized for $R e$ between 100 and 500. So, ideal mixing in relation to liquid-to-gas mass transfer is better approached in the transition region than in other regimes. Moreover, it emerges that increasing $R e$ increases biohydrogen production rate $R_{\max }$ when $R e<100$, while $H_{\max }$ varies only slightly, because this enhances mass transfer rate without impairing the environmental condition of the culture. Conversely, the mechanical stress induced by turbulent eddies reduces $H_{\max }$ even when smaller $t_{m}$, or higher $k_{L} a$ and $R_{\max }$ values are reported for $R e>1000$, which confirms that microorganisms become less efficient.

As a conclusion, both in terms of yield and kinetics, the transitional flow region, $100<R e<1000$, provides the optimum conditions to operate the dark fermentation process in terms of mixing, mass transfer and reaction conditions because mass transfer is the limiting step in the laminar flow region and reaction rate is reached only owing to the supersaturation of dissolved hydrogen in the liquid phase, Conversely, turbulent stress clearly impairs biochemical processes in the turbulent flow region when mass transfer limitation has vanished. This implies that agitation conditions must be adjusted as a function of digestate viscosity to maintain optimum culture conditions, accounting for the variability of substrate composition $v s$. time.

\section{Conclusions}

In this work, experimental analysis of mixing time and liquid-to-gas mass transfer in relation to the hydrogen production potential have been conducted under different medium viscosity and agitation 
conditions in a mechanically-stirred tank reactor equipped with a dual-stage impeller. The following conclusions can be drawn. First, the comparison between the local conductimetric, chemical decolorization, and PLIF methods for the estimation of $t_{m}$ showed that these methods provided close results, but that chemical decolorization may underestimate the mixing time, while PLIF appears as the best trade-off when mixing time becomes small. A master curve expressed as a function of $R e, N_{P}$ and $F o$ was defined, which agreed with literature data for $30<R e<1.1 \cdot 10^{4}: N \cdot t_{m} v s . R e$ fell under laminar flow conditions and became constant when established turbulent flow was achieved. Inter-impeller clearance and tracer injection position influenced $t_{m}$, but the dual-impeller design enhanced mixing in the top region of the tank in comparison to a single-impeller design with the reference configuration, which made possible the addition of the alkaline solution close to the surface in the dark fermentation process with a $\mathrm{pH}$ probe located in the inter-impeller region for $\mathrm{pH}$ control. Then, $\left(k_{L} a\right)_{\mathrm{H}_{2}}$ appeared to be only a function of $R e$ that increased steeply in the transition and turbulent flow regimes. Finally, Reynolds number seems able to reconcile the respective influence of rotation speed and viscosity in order to optimize the dark fermentation process, including mixing time, mass transfer and hydrogen production potential. From the comparison between the characteristic times of mass transfer, mixing and reaction, it arises that hydrogen production was limited by mass transfer under laminar flow, and that the steep decrease in hydrogen production in the turbulent flow regime did not derive from mass transfer rate or mixing time. As a result, the transitional flow region emerged as the optimum condition for dark fermentation, because the $\mathrm{H}_{2}$ production potential was maximized, the maximum production rate was enhanced, ideal mixing was approached for better $\mathrm{pH}$ control, and mass transfer limitation was reduced at the same time.

Concludingly, this work underlines the key role of agitation conditions and digestate viscosity, described univocally as a function of $R e$, on biohydrogen production potential, liquid-to-gas mass transfer and mixing rates, for the scale-up of the dark fermentation process. The consequence is that the change of digestate viscosity induced by the variability of the composition of feed in fermentors must be accounted 
for and counterbalanced by a proper change of agitation conditions so as to maintain optimum biohydrogen production over time.

\section{Acknowledgements}

LABEX IMobS $^{3}$ Innovative Mobility: Smart and Sustainable Solutions, the French National Centre for Scientific Research (CNRS), Auvergne-Rhône-Alpes Regional Council and the European Funds of Regional Development (ERDF/FEDER) are gratefully acknowledged.

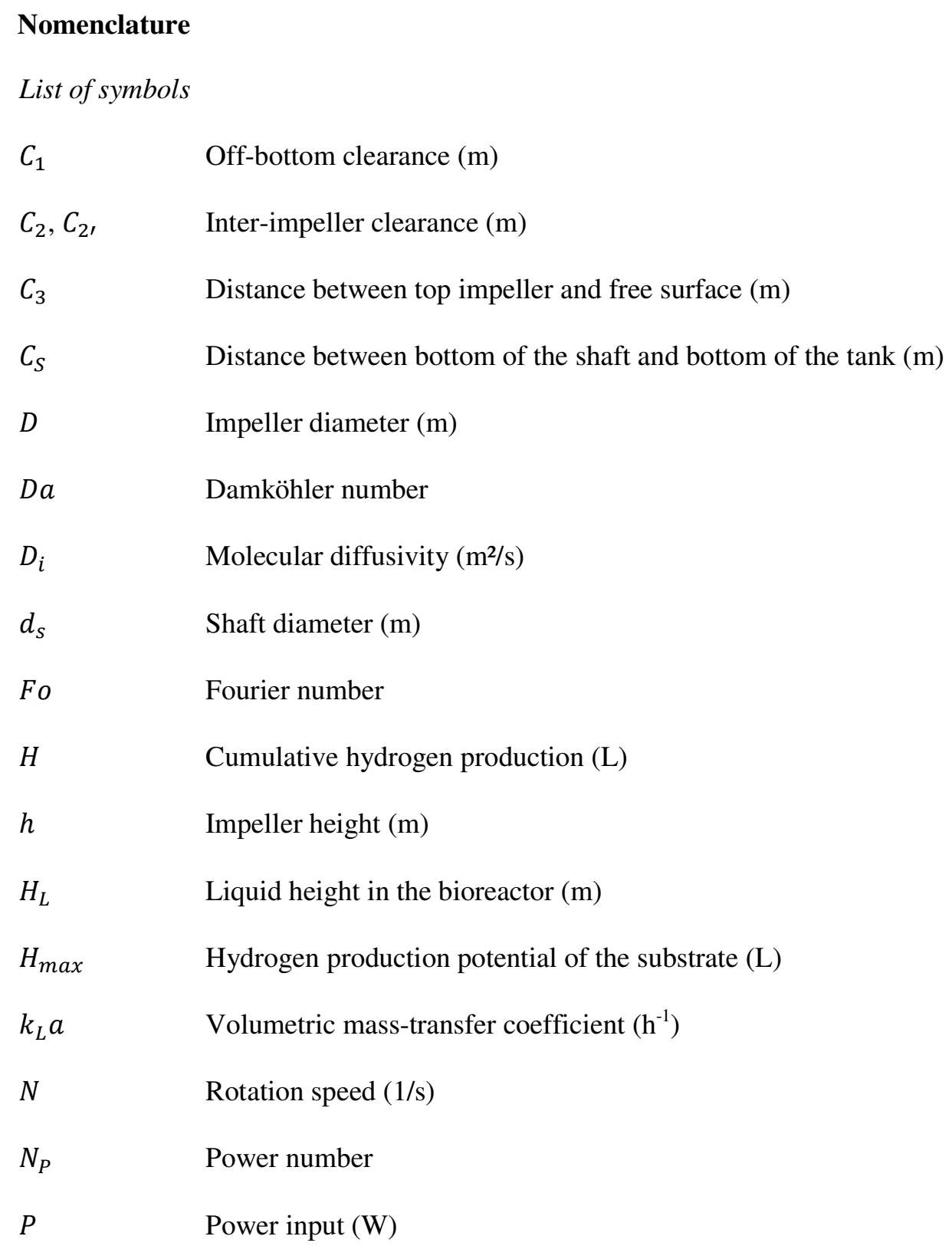




\begin{tabular}{|c|c|}
\hline$P_{I}, P_{I \prime}$ & Injection position of the tracer $(\mathrm{m})$ \\
\hline$R e$ & Reynolds number \\
\hline$R_{\max }$ & Maximum hydrogen production rate $(\mathrm{L} / \mathrm{h})$ \\
\hline$T$ & Bioreactor diameter (m) \\
\hline$t$ & Time (s) \\
\hline$t_{m}$ & Mixing time (s) \\
\hline$V$ & Bioreactor volume $\left(\mathrm{m}^{3}\right)$ \\
\hline \multicolumn{2}{|c|}{ Greek letters } \\
\hline$\lambda$ & Lag time (h) \\
\hline$\mu$ & Dynamic viscosity (Pa.s) \\
\hline$\rho$ & Fluid density $\left(\mathrm{kg} / \mathrm{m}^{3}\right)$ \\
\hline \multicolumn{2}{|c|}{ Abbreviations } \\
\hline$H S V$ & Hue Saturation Value \\
\hline PLIF & Planar Laser Induced Fluorescence \\
\hline VFAs & Volatile Fatty Acids \\
\hline
\end{tabular}

\section{References}

- [1] S.Z. Baykara, Hydrogen: A brief overview on its sources, production and environmental impact, Int. J. Hydrogen Energ. 43 (2018) 10605-10614.

- [2] A. Ghimire, L. Frunzo, F. Pirozzi, E. Trably, R. Escudié, P.N.L. Lens, G. Esposito, A review on dark fermentative biohydrogen production from organic biomass: Process parameters and use of by-products, Appl. Energ. 144 (2015) 73-95.

- [3] A. Noblecourt, G. Christophe, C. Larroche, P. Fontanille, Hydrogen production by dark fermentation from pre-fermented depackaging food wastes, Bioresource Technol. 247 (2018) 864-870.

- [4] G. de Gioannis, M. Friargiu, E. Massi, A. Muntoni, A. Polettini, R. Pomi, D. Spiga, Biohydrogen production from dark fermentation of cheese whey: Influence of pH, Int. J. Hydrogen Energ. 39 (2014) 2093020941. 
- [5] F. Silva-Illanes, E. Tapia-Venegas, M.C. Schiappacasse, E. Trably, G. Ruiz-Filippi, Impact of hydraulic retention time (HRT) and pH on dark fermentative hydrogen production from glycerol, Energy. 141 (2017) 358-367.

- [6] E. Elbeshbishy, B.R. Dhar, G. Nakhla, H.S. Lee, A critical review on inhibition of dark biohydrogen fermentation, Renew. Sust. Energ. Rev. 79 (2017) 656-668.

- [7] M.C. Castro-Villalobos, J.L. Garcia-Morales, F.J. Fernandez, By-products inhibition effects on biohydrogen production, Int. J. Hydrogen Energ. 37 (2012) 7077-7083.

- [8] B. Mandal, K. Nath, D. Das, Improvement of biohydrogen production under decreased partial pressure of $\mathrm{H}_{2}$ by Enterobacter cloacae, Biotechnol. Lett. 28 (2006) 831-835.

- [9] J.R. Bastidas-Oyanedel, Z. Mohd-Zaki, R.J. Zeng, N. Bernet, S. Pratt, J.P. Steyer, D.J. Batstone, Gas controlled hydrogen fermentation, Bioresource Technol. 110 (2012) 503-509.

- [10] K.S. Lee, T.S. Tseng, Y.W. Liu, Y.D. Hsiao, Enhancing the performance of dark fermentative hydrogen production using a reduced pressure fermentation strategy, Int. J. Hydrogen Energ. 37 (2012) 1555615562.

- [11] M.A.Z. Bundhoo, R. Mohee, Inhibition of dark fermentative bio-hydrogen production: A review, Int. J. Hydrogen Energ. 41 (2016) 6713-6733.

- [12] J. Rodriguez, R. Kleerebezem, J.M. Lema, M.C. van Loosdrecht, Modeling product formation in anaerobic mixed culture fermentation, Biotechnol. Bioeng. 93 (2006) 592-606.

- [13] Y. Zhang, F. Zhang, M. Chen, P.N. Chu, J. Ding, R.J. Zeng, Hydrogen supersaturation in extremethermophilic $\left(70^{\circ} \mathrm{C}\right)$ mixed culture fermentation, Appl. Energ. 109 (2013) 213-219.

- [14] B. Chezeau, Ch. Vial, Combined effects of digestate viscosity and agitation conditions on the fermentative biohydrogen production, Biochem. Eng. J. 142 (2019) 105-116.

- [15] J. Lindmark, E. Thorin, R. Bel Fdhila, E. Dahlquist, Effect of mixing on the result of anaerobic digestion: Review, Renew. Sust. Energ. Rev. 40 (2014) 1030-1047.

- [16] B. Ruggeri, T. Tommasi, Efficiency and efficacy of pre-treatment and bioreaction for bio- $\mathrm{H}_{2}$ energy production from organic waste, Int. J. Hydrogen Energ. 37 (2012) 6491-6502.

- [17] Z. Trad, C. Vial, J.P. Fontaine, C. Larroche, Mixing and liquid-to-gas mass transfer under digester operating conditions, Chem. Eng. Sci. 170 (2017) 606-627. 
- [18] L. Vega-Alvarado, B. Taboada, A. Hidalgo-Millan, G. Ascanio, Image analysis method for the measurement of mixing times in stirred vessels, Chem. Eng. Technol. 34 (2011) 859-866.

- [19] F. Cabaret, S. Bonnot, L. Fradette, P.A. Tanguy, Mixing time analysis using colorimetric methods and image processing, Ind. Eng. Chem. Res. 46 (2007) 5032-5042.

- [20] G. Ascanio, Mixing time in stirred vessels: A review of experimental techniques, Chinese J. Chem. Eng. 23 (2015) 1065-1076.

- [21] R.R. Hemrajani, G.B. Tatterson, Mechanically stirred vessels. In Handbook of Industrial Mixing: Science and Practice ( $1^{\text {st }}$ edition) (chapter 6). Editor: E.L. Paul, V.A. Atiemo-Obeng, S.M. Kresta. John Wiley and Sons, Inc. Hoboken, NJ, USA, 2004.

- [22] A. Busciglio, G. Montante, A. Paglianti, Flow field and homogenization time assessment in continuously-fed stirred tanks, Chem. Eng. Res. Des. 102 (2015) 42-56.

- [23] M. Giona, A. Paglianti, S. Cerbelli, S. Pintus, A. Adrover, Dispersion in stirred tank reactor: Asymptotic properties and mixing characterization, Can. J. Chem. Eng. 80 (2002) 580-590.

- [24] I. Bouwmans, A. Barker, H.E.A. van Den Akker, Blending liquids of differing viscosities and densities in stirred vessels, Chem. Eng. Res. Des. 75 (1997) 777-783.

- [25] M.M. Alvarez, P.E. Arratia, F.J. Muzzio, Laminar mixing in eccentric stirred tank systems, Can. J. Chem. Eng. 80 (2002) 546-557.

- [26] G. Ascanio, S. Foucault, P.A. Tanguy, Time-periodic mixing of shear-thinning fluids, Chem. Eng. Res. Des. 82 (2004) 1199-1203.

- [27] K. Stamatopoulos, F. Alberini, H. Batchelor, M.J.H. Simmons, Use of PLIF to assess the mixing performance of small volume USP 2 apparatus in shear thinning media, Chem. Eng. Sci. 145 (2016) 1-9.

- [28] J.M.T. Vasconcelos, J.M. Barata, S.S. Alves, Transitional mixing in multiple-turbine agitated tanks, Chem. Eng. J. Bioch. Eng. 63 (1996) 53-58.

- [29] M.L. Collignon, Etude du mélange par P.I.V. et P.L.I.F. dans un bioréacteur de culture cellulaire. (In french). (2007)._http://hdl.handle.net/2268/5008.

- [30] Z. Trad, J.P. Fontaine, C. Larroche, C. Vial, Experimental and numerical investigation of hydrodynamics and mixing in a dual-impeller mechanically-stirred digester, Chem. Eng. Sci. 329 (2017) 142-155. 
- [31] A.W. Nienow, On impeller circulation and mixing effectiveness in the turbulent flow regime, Chem. Eng. Sci. 52 (1997) 2557-2565.

- [32] R.K. Grenville, A.W. Nienow, Blending of miscible liquids. In Handbook of Industrial Mixing: Science and Practice ( $1^{\text {st }}$ edition) (chapter 9). Editor: E.L. Paul, V.A. Atiemo-Obeng, S.M. Kresta. John Wiley and Sons, Inc. Hoboken, NJ, USA, 2004.

- [33] A.A. Yawalkar, A.B.M. Heesink, G.F. Versteeg, V.G. Pangarkar, Gas-liquid mass transfer coefficient in stirred tank reactors, Can. J. Chem. Eng. 80 (2008) 840-848. 

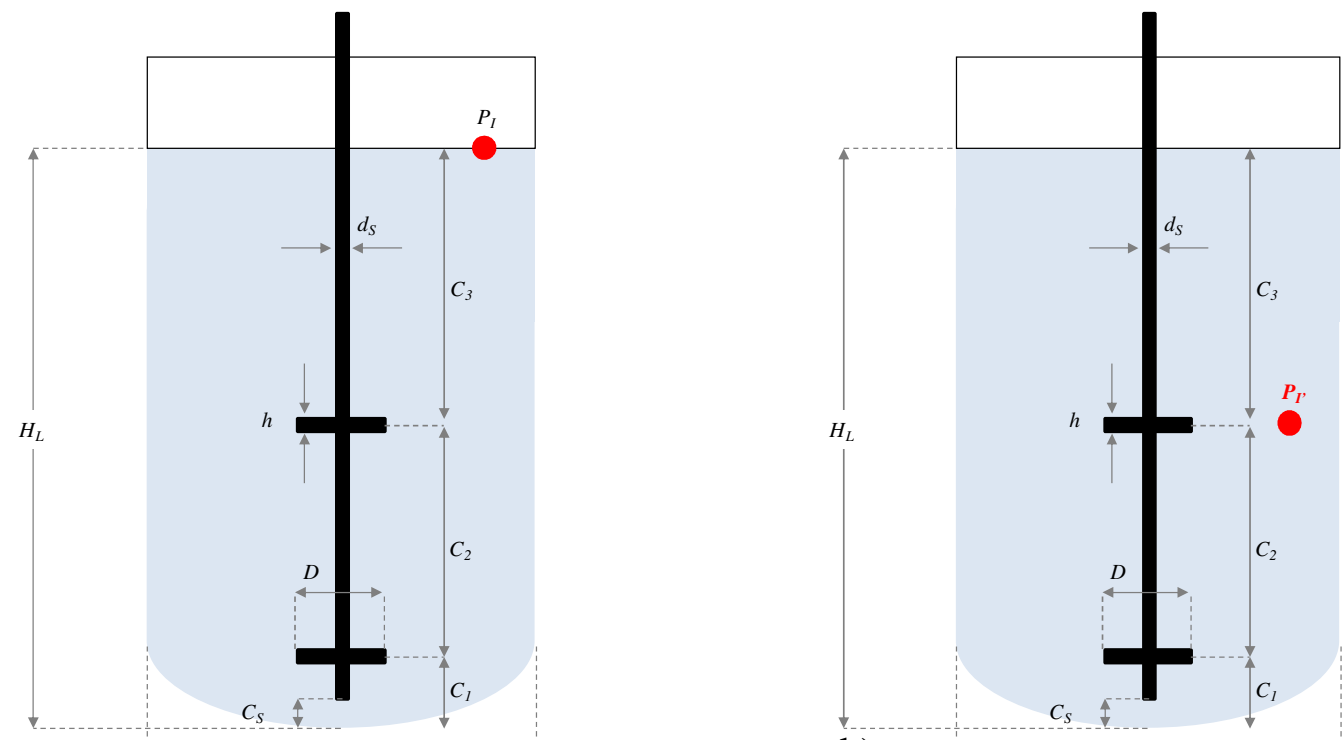

a)

b)
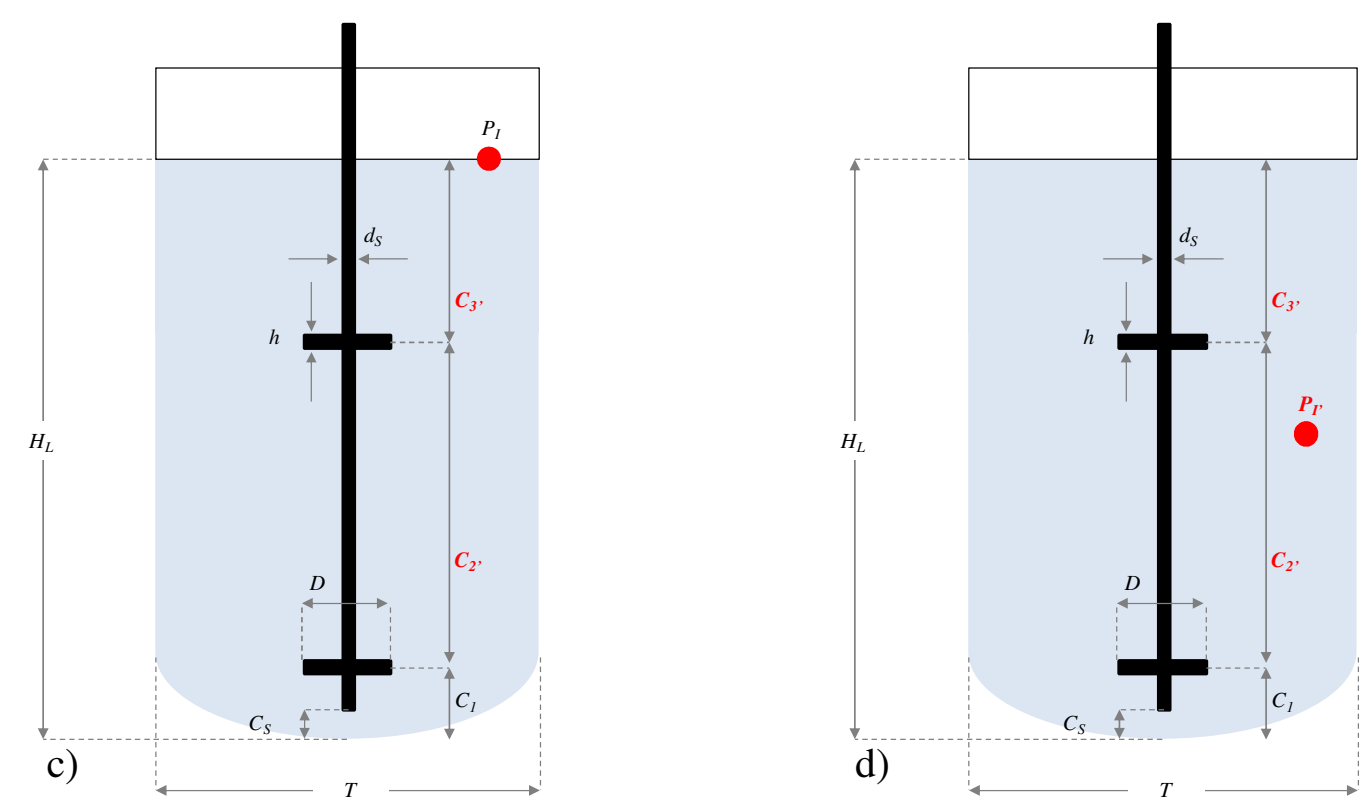

d)

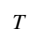

Fig. 1. Sketch of the impeller design of the reference configuration (a) and the alternative configurations $1(b), 2(c)$ and $3(d)$. 

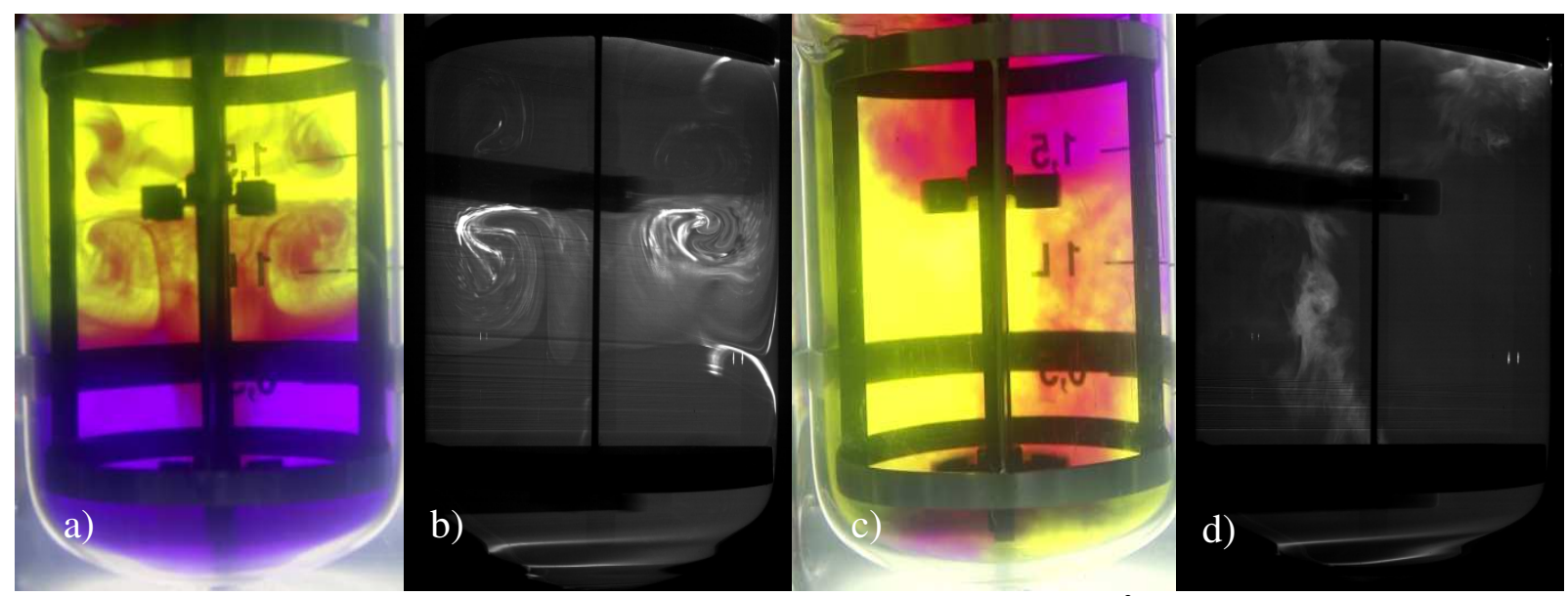

Fig. 2. Flow structure obtained under laminar flow at $R e=33\left(N=40 \mathrm{rpm}\right.$ and $\mu=6.1 \cdot 10^{-2}$ Pa.s $)$ after $60 \mathrm{~s}$ mixing with the chemical decolorization (a) and PLIF methods (b), respectively; under turbulent flow at Re $=1.1 \cdot 10^{4}\left(\mathrm{~N}=200 \mathrm{rpm}\right.$ and $\left.\mu=9.0 \cdot 10^{-4} \mathrm{~Pa} . \mathrm{s}\right)$ after $2 \mathrm{~s}$ of mixing with the chemical decolorization (c) and PLIF methods $(d)$, respectively. 

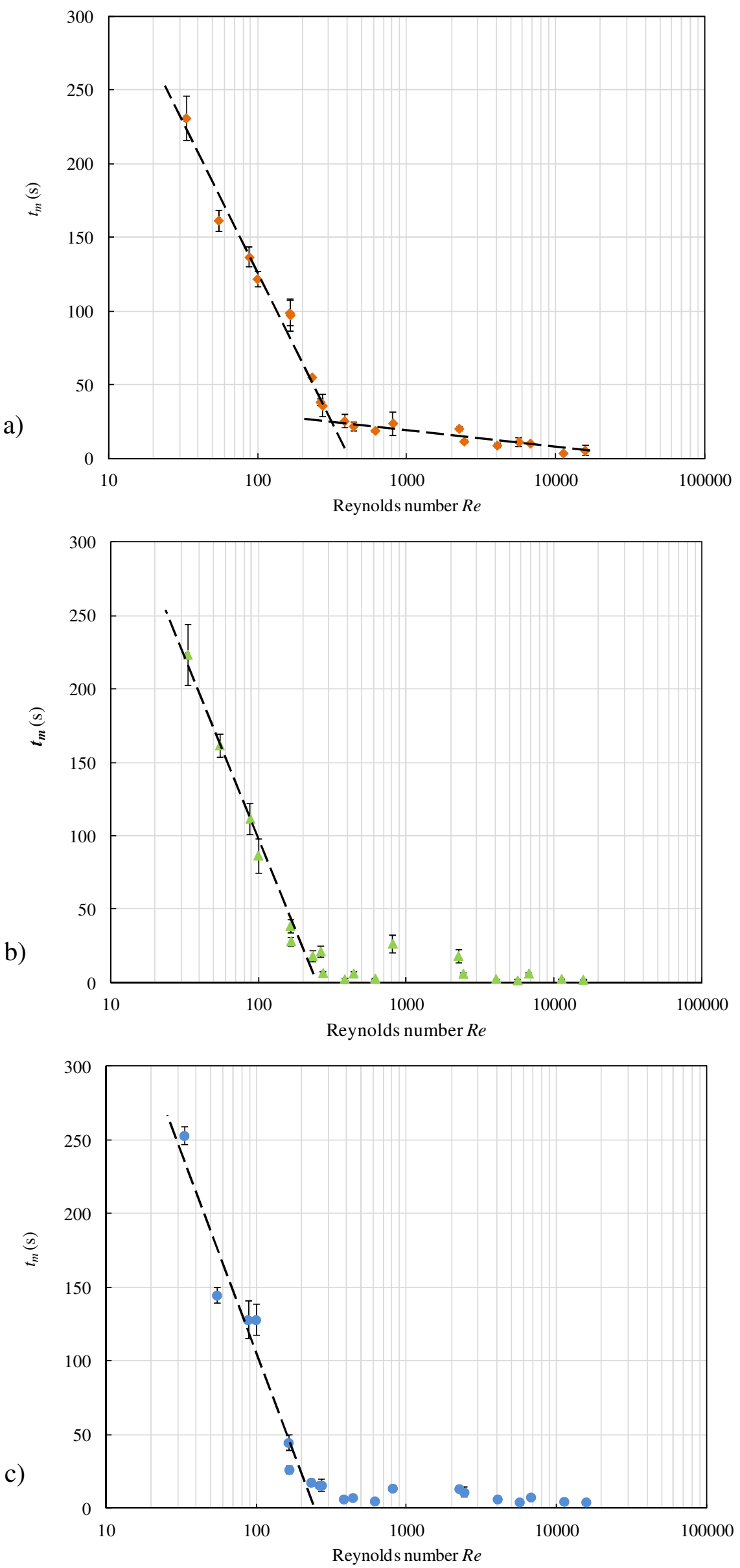

Fig. 3. Experimental mixing time versus Reynolds number using the conductimetric (a), chemical decolorization (b) and PLIF methods (c). Error bars are based on triplicate experiments. 
a)

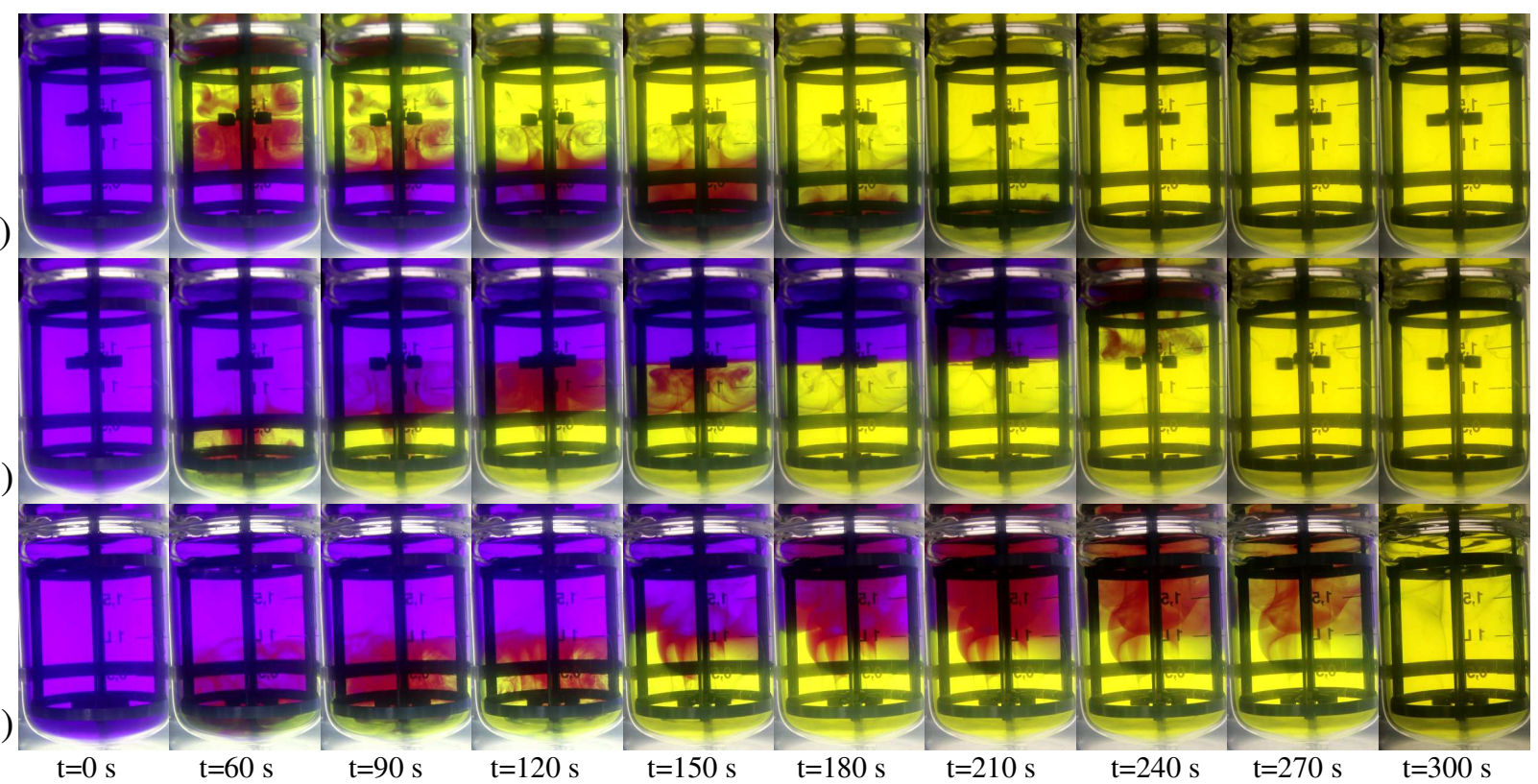

Fig. 4. Mixing pattern obtained under laminar flow at $R e=33\left(N=40 \mathrm{rpm}\right.$ and $\mu=6.1 \cdot 10^{-2}$ Pa.s) for the reference configuration (a) and the alternative configurations $1(b)$ and $2(c)$. 

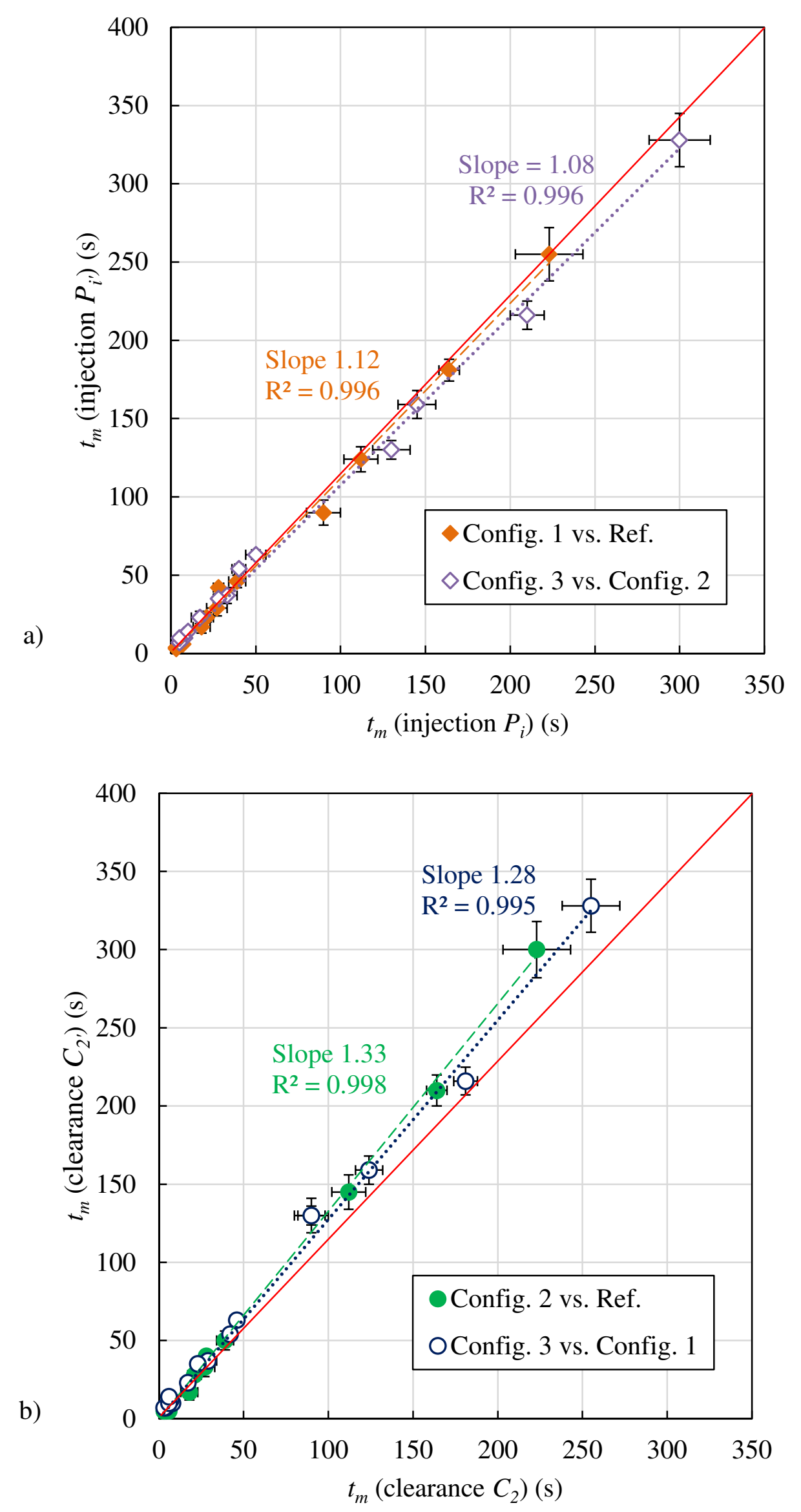

Fig. 5. Comparison of experimental mixing time between configuration 1 and the reference design, and between configuration 3 and 2 (a); comparison of experimental mixing time between configuration 2 and the reference design, and between configuration 3 and $1(b)$. Error bars are based on triplicate experiments. 

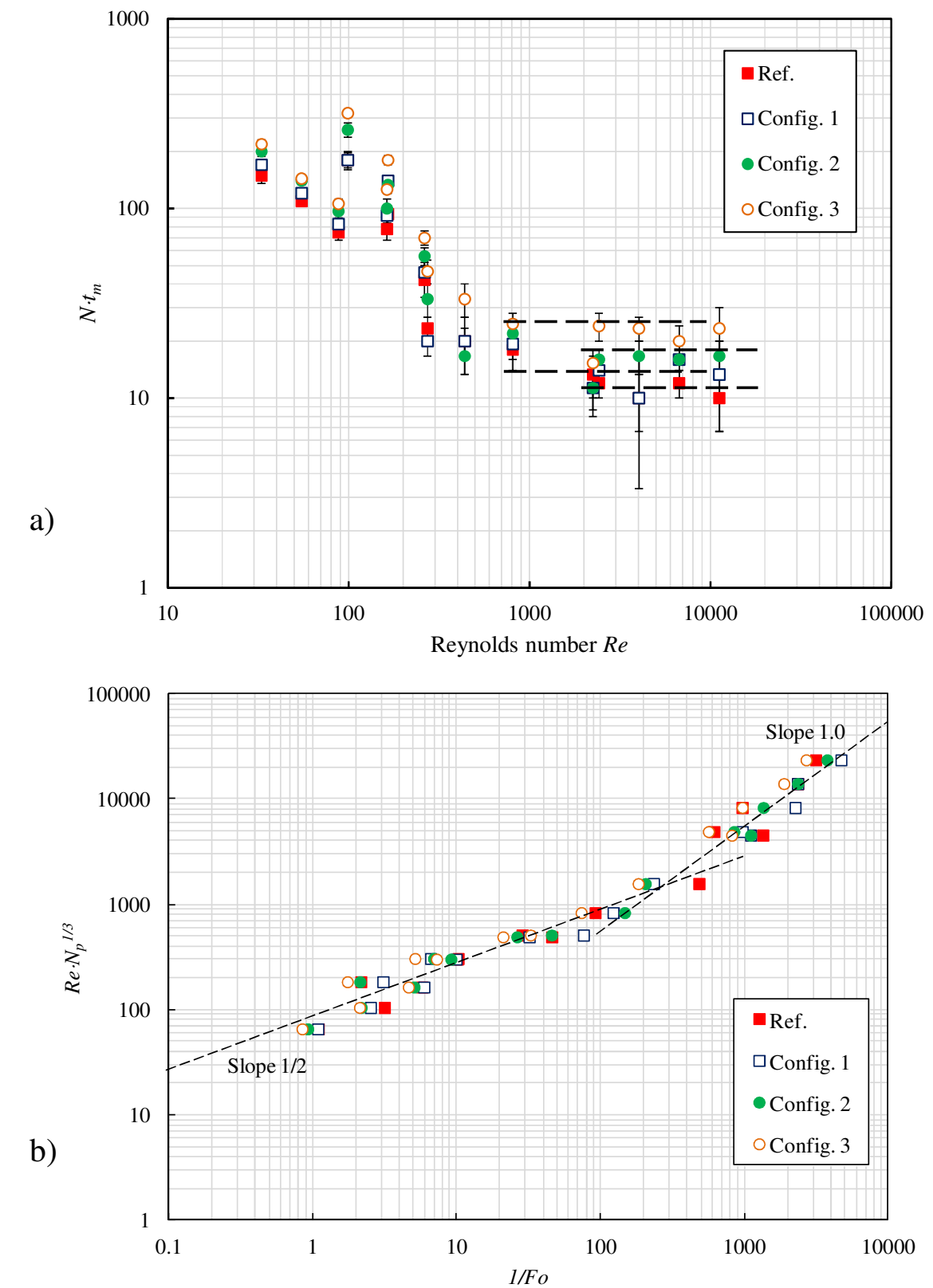

Fig. 6. Dimensionless mixing time $N t_{m}$ versus $R e(a) R e \cdot N_{p}^{1 / 3}$ plot versus $1 / F o(b)$ for the four configurations. Error bars are based on triplicate experiments. 


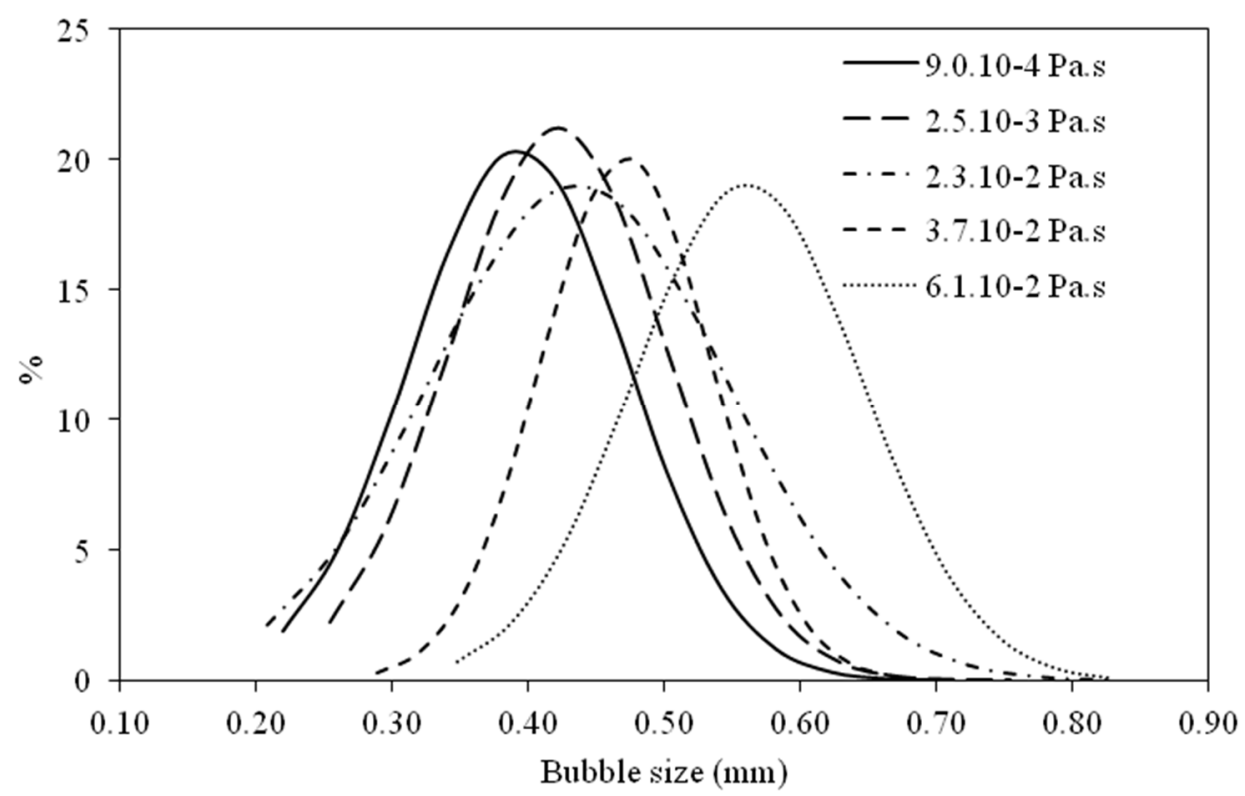

Fig. 7. Fitted bubble size distribution with different medium viscosity $(N=120 \mathrm{rpm})$. 

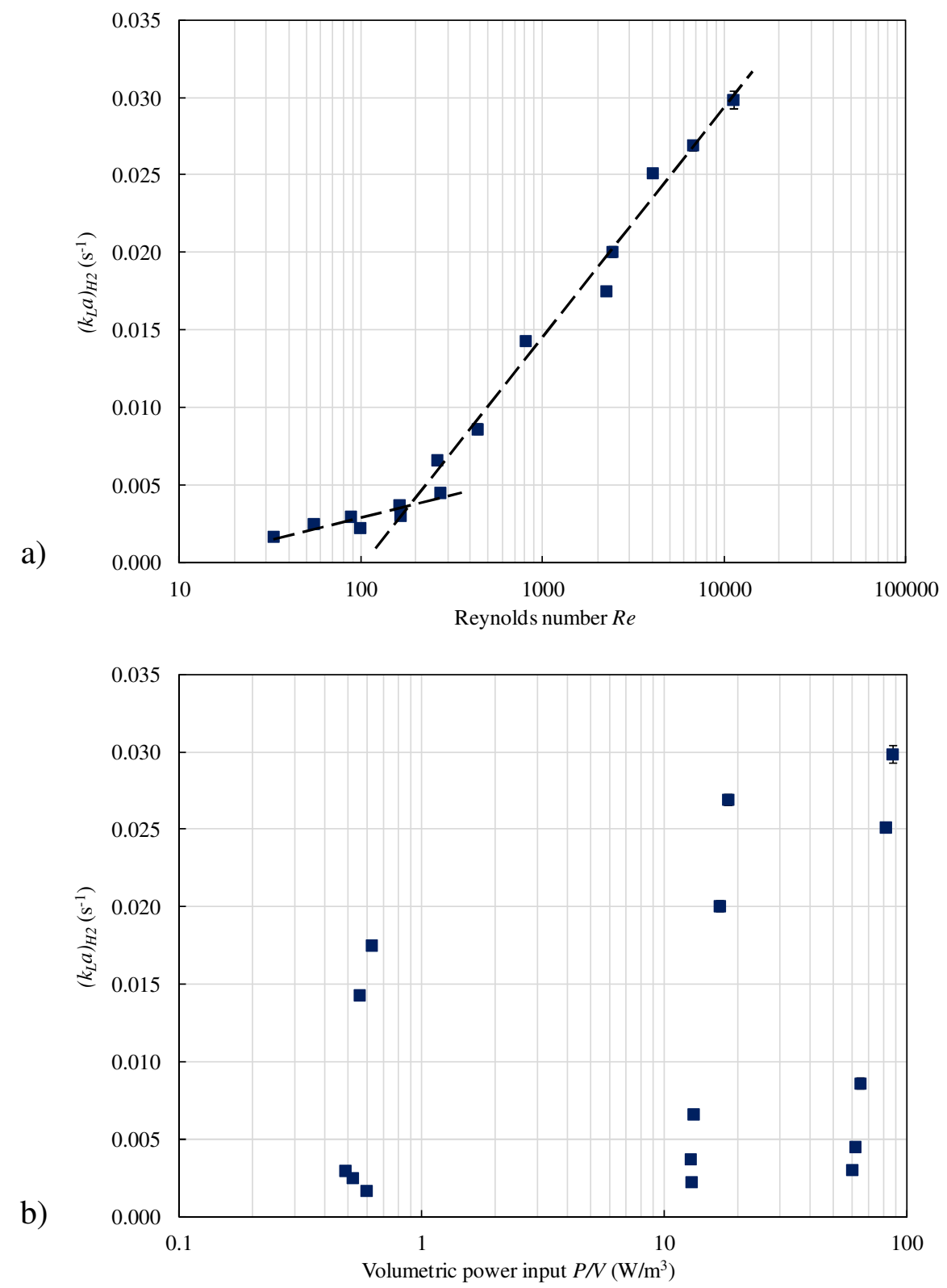

Fig. 8. Experimental volumetric mass transfer coefficient versus Re (a) and volumetric power input (b). Error bars are based on triplicate experiments. 

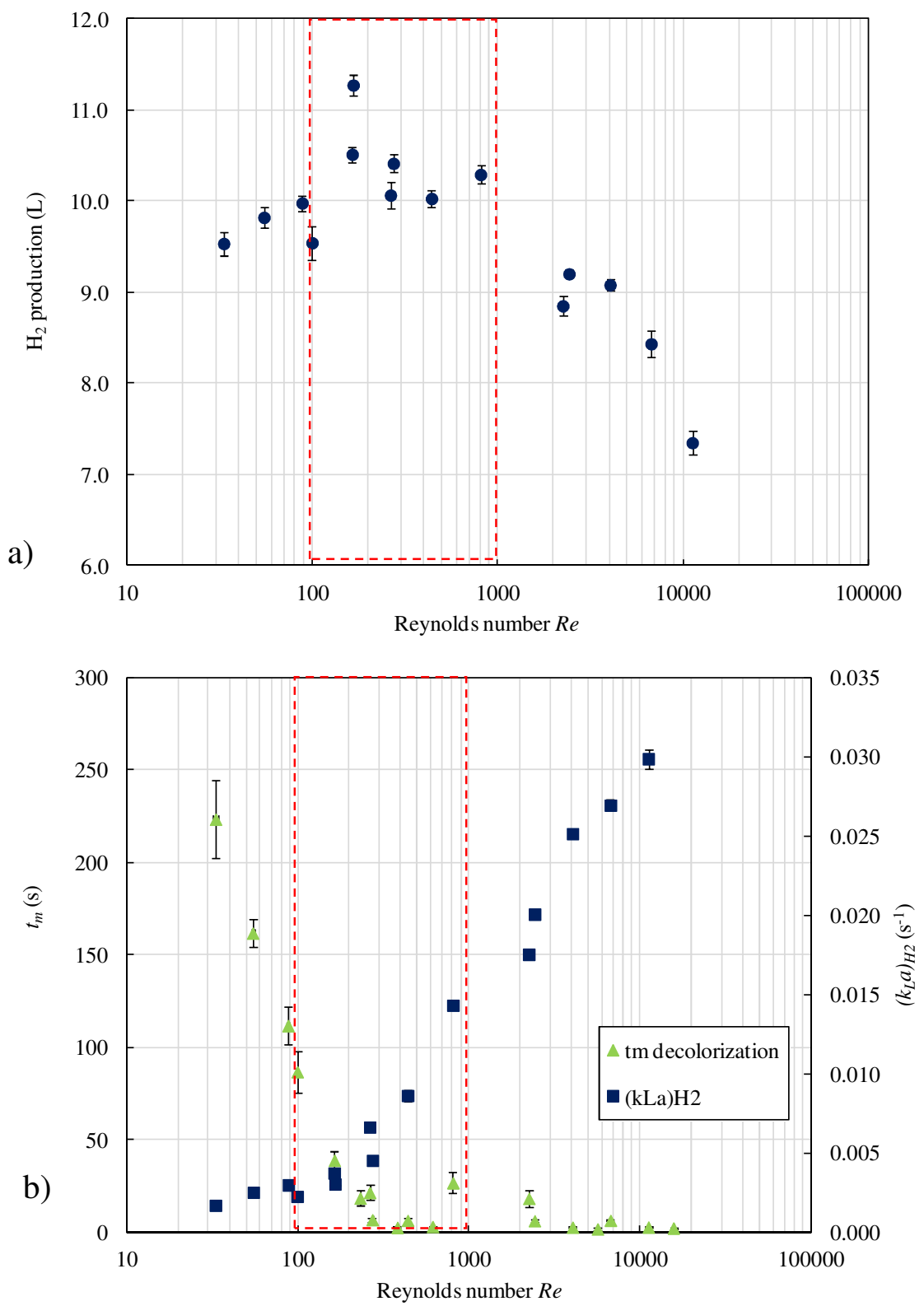

Fig. 9. Experimental hydrogen production potential versus $R e(a)$, and experimental volumetric mass transfer coefficient and mixing time versus $R e(b)$. Error bars are based on triplicate experiments. 


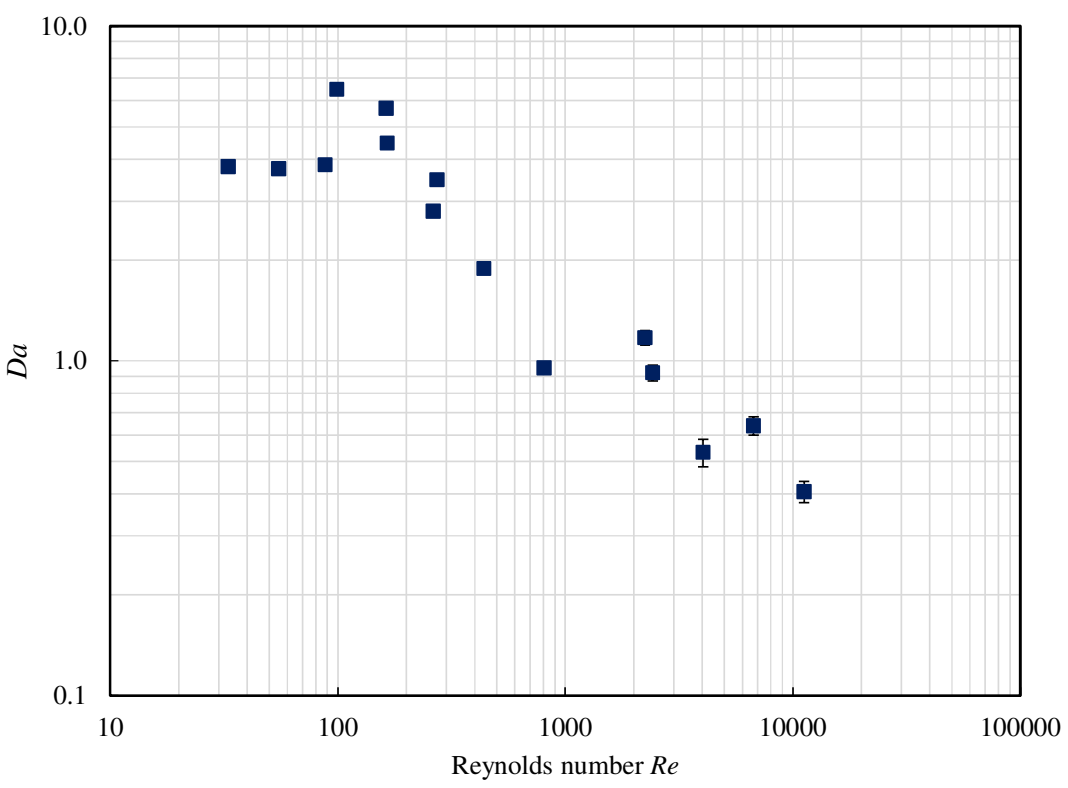

Fig.10. Damköhler number evolution versus Reynolds number. Error bars are based on triplicate experiments. 
Table 1. Advantages and limitations of the conductimetric, chemical decolorization and PLIF methods (adapted from Ascanio [20]).

\begin{tabular}{|c|c|c|}
\hline Techniques & Advantages & Limitations \\
\hline PLIF & $\begin{array}{l}\text { Non-intrusive } \\
\text { Direct measurement } \\
\text { Visualisation of 2-D isolated mixing regions } \\
\text { Reveal flow structure } \\
\text { Possible with low aerated conditions } \\
\end{array}$ & $\begin{array}{l}\text { Expensive (camera and laser) } \\
\text { Only for transparent systems } \\
\text { Calibration prior to the experiments } \\
\text { Limited applicability under aerated conditions }\end{array}$ \\
\hline $\begin{array}{l}\text { Chemical } \\
\text { Decolorization }\end{array}$ & $\begin{array}{l}\text { Non-intrusive } \\
\text { Direct measurement } \\
\text { Visualisation of 2-D isolated mixing regions } \\
\text { Reveal flow structure } \\
\text { No calibration } \\
\text { Possible under aerated or unaerated conditions }\end{array}$ & $\begin{array}{l}\text { Only for transparent systems } \\
\text { Limited applicability under highly aerated } \\
\text { conditions in turbulent regime } \\
\text { Temporal resolution with image processing } \\
\text { techniques }\end{array}$ \\
\hline Conductimetric & $\begin{array}{l}\text { Direct measurement } \\
\text { For transparent and opaque systems } \\
\text { Possible under aerated or unaerated conditions }\end{array}$ & $\begin{array}{l}\text { Intrusive and invasive } \\
\text { Calibration prior to the experiments } \\
\text { Mixing time depends on the position of the probe } \\
\text { Sensitive to temperature changes and no flow } \\
\text { structure }\end{array}$ \\
\hline
\end{tabular}


Table 2. Dynamic viscosity of the solution as a function of the percentage of polyalkylene glycol.

\begin{tabular}{cc}
\hline \% PAG (v/v) & Viscosity (Pa.s) \\
\hline 0 & $9.0 \cdot 10^{-4}$ \\
5 & $2.5 \cdot 10^{-3}$ \\
10 & $2.3 \cdot 10^{-2}$ \\
20 & $3.7 \cdot 10^{-2}$ \\
30 & $6.1 \cdot 10^{-2}$ \\
\hline
\end{tabular}


Table 3. Comparison of mixing time data from several techniques with the reference configuration.

\begin{tabular}{|c|c|c|c|c|c|}
\hline \multirow[b]{2}{*}{$\begin{array}{c}\text { Rotation speed } \\
\qquad N \text { (rpm) }\end{array}$} & \multirow[b]{2}{*}{$\begin{array}{c}\text { Dynamic } \\
\text { viscosity } \mu \\
\text { (Pa.s) }\end{array}$} & \multirow[b]{2}{*}{$\begin{array}{c}\text { Reynolds } \\
\text { number } R e\end{array}$} & \multicolumn{3}{|c|}{ Mixing time $t_{m}(\mathrm{~s})$} \\
\hline & & & $\begin{array}{l}\text { Conductimetric } \\
\text { technique }\end{array}$ & $\begin{array}{c}\text { Chemical } \\
\text { decolorization } \\
\text { technique }\end{array}$ & $\begin{array}{l}\text { PLIF } \\
\text { technique }\end{array}$ \\
\hline 40 & $9.0 \cdot 10^{-4}$ & 2240 & $20 \pm 1$ & $18 \pm 5$ & $14 \pm 2$ \\
\hline 40 & $2.5 \cdot 10^{-3}$ & 807 & $24 \pm 8$ & $27 \pm 6$ & $14 \pm 1$ \\
\hline 40 & $2.3 \cdot 10^{-2}$ & 88 & $140 \pm 7$ & $110 \pm 10$ & $130 \pm 10$ \\
\hline 40 & $3.7 \cdot 10^{-2}$ & 55 & $160 \pm 8$ & $164 \pm 6$ & $145 \pm 5$ \\
\hline 40 & $6.1 \cdot 10^{-2}$ & 33 & $230 \pm 20$ & $220 \pm 20$ & $253 \pm 6$ \\
\hline 120 & $9.0 \cdot 10^{-4}$ & 6722 & $10 \pm 1$ & $6 \pm 1$ & $8 \pm 1$ \\
\hline 120 & $2.5 \cdot 10^{-3}$ & 2420 & $12 \pm 1$ & $6 \pm 1$ & $11 \pm 3$ \\
\hline 120 & $2.3 \cdot 10^{-2}$ & 263 & $38 \pm 3$ & $21 \pm 4$ & $16 \pm 2$ \\
\hline 120 & $3.7 \cdot 10^{-2}$ & 163 & $99 \pm 9$ & $40 \pm 5$ & $45 \pm 5$ \\
\hline 120 & $6.1 \cdot 10^{-2}$ & 99 & $122 \pm 6$ & $90 \pm 10$ & $130 \pm 10$ \\
\hline 200 & $9.0 \cdot 10^{-4}$ & 11204 & $4 \pm 1$ & $3 \pm 1$ & $5 \pm 1$ \\
\hline 200 & $2.5 \cdot 10^{-3}$ & 4033 & $9 \pm 1$ & $3 \pm 1$ & $7 \pm 2$ \\
\hline 200 & $2.3 \cdot 10^{-2}$ & 438 & $22 \pm 3$ & $6 \pm 2$ & $8 \pm 2$ \\
\hline 200 & $3.7 \cdot 10^{-2}$ & 273 & $36 \pm 8$ & $7 \pm 1$ & $16 \pm 4$ \\
\hline 200 & $6.1 \cdot 10^{-2}$ & 165 & $100 \pm 10$ & $28 \pm 3$ & $27 \pm 3$ \\
\hline
\end{tabular}


Table 4. Characteristic time scales and dimensionless parameters derived from this work.

\begin{tabular}{cccccccccccc}
\hline $\boldsymbol{R} \boldsymbol{e}$ & $\boldsymbol{N}(\mathbf{r p m})$ & $\boldsymbol{\mu}(\mathbf{P a . s})$ & $\boldsymbol{t}_{\boldsymbol{m}}(\boldsymbol{s})$ & $\boldsymbol{N} \cdot \boldsymbol{t m}$ & $\mathbf{1} / \boldsymbol{k}_{\boldsymbol{L}} \boldsymbol{a}(\mathbf{s})$ & $\boldsymbol{\lambda}(\mathbf{h})$ & $\begin{array}{c}\boldsymbol{R}_{\max } \\
(\mathbf{L} / \mathbf{h})\end{array}$ & $\boldsymbol{H}_{\max }(\mathbf{L})$ & $\boldsymbol{k}_{\boldsymbol{L}} \boldsymbol{a} \cdot \boldsymbol{t}_{\boldsymbol{m}}$ & $\boldsymbol{D a}$ \\
\hline 33 & 40 & $6.1 \cdot 10^{-2}$ & 223 & 149 & 593 & 16.9 & 0.73 & 10.1 & 0.38 & 3.8 \\
55 & 40 & $3.7 \cdot 10^{-2}$ & 164 & 109 & 398 & 11.3 & 1.07 & 10.2 & 0.41 & 3.8 \\
88 & 40 & $2.3 \cdot 10^{-2}$ & 112 & 75 & 335 & 10.5 & 1.30 & 10.2 & 0.33 & 3.9 \\
99 & 120 & $6.1 \cdot 10^{-2}$ & 90 & 180 & 444 & 14.3 & 1.65 & 10.7 & 0.20 & 6.5 \\
163 & 120 & $3.7 \cdot 10^{-2}$ & 39 & 78 & 268 & 14.5 & 2.39 & 11.1 & 0.15 & 5.7 \\
165 & 200 & $6.1 \cdot 10^{-2}$ & 28 & 93 & 329 & 12.9 & 1.54 & 12.3 & 0.09 & 4.5 \\
263 & 120 & $2.3 \cdot 10^{-2}$ & 21 & 42 & 151 & 11.1 & 2.10 & 10.7 & 0.14 & 2.8 \\
273 & 200 & $3.7 \cdot 10^{-2}$ & 7 & 23 & 221 & 12.9 & 1.77 & 11.4 & 0.03 & 3.5 \\
438 & 200 & $2.3 \cdot 10^{-2}$ & 6 & 20 & 116 & 11.1 & 1.84 & 10.9 & 0.05 & 1.9 \\
807 & 40 & $2.5 \cdot 10^{-3}$ & 27 & 18 & 70 & 10.3 & 1.54 & 10.5 & 0.39 & 1.0 \\
2240 & 40 & $9.0 \cdot 10^{-4}$ & 18 & 12 & 57 & 10.7 & 2.32 & 8.9 & 0.32 & 1.2 \\
2420 & 120 & $2.5 \cdot 10^{-3}$ & 6 & 12 & 50 & 11.1 & 2.09 & 9.6 & 0.12 & 0.9 \\
4033 & 200 & $2.5 \cdot 10^{-3}$ & 3 & 10 & 40 & 10.5 & 1.51 & 10.2 & 0.08 & 0.5 \\
6722 & 120 & $9.0 \cdot 10^{-4}$ & 6 & 12 & 37 & 10.8 & 1.95 & 8.8 & 0.16 & 0.6 \\
11204 & 200 & $9.0 \cdot 10^{-4}$ & 3 & 10 & 33 & 10.3 & 1.37 & 8.0 & 0.09 & 0.4 \\
\hline
\end{tabular}

